\title{
Synthesis and analytical characterization of new thiazol-2-(3H)-ones as human neutrophil elastase (HNE) inhibitors
}

\author{
Letizia Crocetti ${ }^{1}$, Gianluca Bartolucci ${ }^{1}$, Agostino Cilibrizzi ${ }^{2}$, Maria Paola Giovannoni ${ }^{1 *}$, Gabriella Guerrini ${ }^{1}$, \\ Antonella lacovone ${ }^{1}$, Marta Menicatti ${ }^{1}$, Igor A. Schepetkin ${ }^{3}$, Andrei I. Khlebnikov ${ }^{4,5}$, Mark T. Quinn ${ }^{3}$ \\ and Claudia Vergelli ${ }^{1}$
}

\begin{abstract}
Human neutrophil elastase (HNE) is a potent serine protease belonging to the chymotrypsin family and is involved in a variety of pathologies affecting the respiratory system. Thus, compounds able to inhibit HNE proteolytic activity could represent effective therapeutics. We present here the synthesis of new thiazol-2-(3H)-ones as an elaboration of potent HNE inhibitors with an isoxazol-5-(2H)-one scaffold that we recently identified. Two-dimensional NMR spectroscopic techniques and tandem mass spectrometry allowed us to correctly assign the structure of the final compounds arising from both tautomers of the thiazol-2-(3H)-one nucleus ( $\mathrm{N}-3$ of the thiazol-2-(3H)-one and $3-\mathrm{OH}$ of the thiazole). All new compounds were tested as HNE inhibitors, and no activity was found at the highest concentration used $(40 \mu \mathrm{M})$, demonstrating that the thiazol-2-(3H)-one is not a good scaffold for HNE inhibitors. Molecular modelling experiments indicate that the low-energy pose might limit the nucleophilic attack on the endocyclic carbonyl group of the thiazolone-based compounds by HNE catalytic Ser195, in contrast to isoxazol-5-(2H)-one analogues.
\end{abstract}

Keywords: Thiazol-2-(3H)-one, Synthesis, LC-MS/MS, ERMS, Human neutrophil elastase

\section{Introduction}

Human neutrophil elastase (HNE) is a small, soluble glycoprotein of about $30 \mathrm{kDa}$ belonging to the chymotrypsin family of serine proteases [1] and is expressed primarily in neutrophils, but also in monocytes and mast cells [2]. HNE plays an important role in the maintenance of tissue homeostasis and repair due to its proteolytic action on structural proteins [2,3]. HNE performs its proteolytic action through a catalytic triad consisting of Ser195Asp102-His57, where the powerful nucleophile oxygen of Ser195 attacks the carbonyl carbon involved in the peptide bond $[4,5]$. HNE is involved in a variety of pathologies affecting the respiratory system, such as chronic obstructive pulmonary disease (COPD), acute respiratory distress syndrome (ARDS), acute lung injury (ALI)

\footnotetext{
*Correspondence: mariapaola.giovannoni@unifi.it

1 Sezione di Farmaceutica e Nutraceutica, NEUROFARBA, Università degli Studi di Firenze, Via Ugo Schiff 6, 50019 Sesto Fiorentino, Firenze, Italy Full list of author information is available at the end of the article
}

and cystic fibrosis (CF) [6-8]. Currently, Sivelestat, which is used for treatment of ALI and ARDS ([9], Fig. 1), and Prolastin, which is used in the therapy of $\alpha 1$-antitripsin deficiency (AATD) [10], are the only HNE inhibitors commercially available. Moreover, Alvelestat (AZD9668, Fig. 1) [11, 12] and Bay 85-8501 [13] are two promising compounds in phase II clinical trials for the treatment of COPD and CF (Fig. 1).

Our research group is involved in the design and synthesis of small molecules with HNE inhibitory activity, and we have identified a number of new classes of inhibitors based on different bicyclic scaffolds [14-17]. The most potent compounds are $N$-benzoylindazole derivatives, which have $\mathrm{IC}_{50}$ values in the low nanomolar range $\left(\mathrm{IC}_{50}=7-80 \mathrm{nM}\right)[14,16]$. Moreover, we recently reported new isoxazolone-based derivatives with $\mathrm{HNE}$ inhibitory activity in the low nanomolar range $\left(\mathrm{IC}_{50}=20-96 \mathrm{nM}\right)$ [18]. Molecular modeling studies on this class of compounds allowed to establish which carbonyl group was involved in the attack of Ser195 (i.e., 
<smiles>CC(C)(C)C(=O)Oc1ccc(S(=O)(=O)Nc2ccccc2C(=O)NCC(=O)O[Na])cc1</smiles><smiles>Cc1c(-c2ccnn2C)cc(C(=O)NCc2ccc(S(C)(=O)=O)cn2)c(=O)n1-c1cccc(C(F)(F)F)c1</smiles>

Alvelestat (AZD9668) $\mathrm{IC}_{50}=12 \mathrm{nM}$<smiles>CC1=C(C#N)[C@@H](c2ccc(C#N)cc2S(C)(=O)=O)N(C)C(=O)N1c1cccc(C(F)(F)F)c1</smiles>

BAY 85-8501

$\mathrm{IC}_{50}=0.065 \mathrm{nM}$

Fig. 1 HNE inhibitors

HNE catalytic residue) and define the endocyclic carbonyl at position 5 of the isoxazolone core as a critical requirement for inhibitory activity [18]. Starting from these results, we report here the development and analytical characterization of a new series of heterocyclic compounds based on the thiazol-2-(3H)-one scaffold originally designed as possible HNE inhibitors (Fig. 2).

\section{Chemistry}

All compounds were synthesized as reported in Schemes 1, 2, 3, and the structures were univocally confirmed on the basis of analytical and spectral data, such as two-dimensional NMR spectroscopic techniques (i.e. HSQC and HMBC, see Additional file 1) and tandem mass spectrometry. According to literature on thiazol2 - $(3 H)$-ones, the predominant isomer in solution seems to be the 2-oxo form [19], although the alkylation reactions can take place on both tautomers (i.e., N-3 of the thiazol-2-(3H)-one and 3-OH of the thiazole) [20].

Scheme 1 shows the synthetic pathways to obtain final compounds $2 \mathbf{a}-\mathbf{f}$, and $3 \mathbf{a}-\mathbf{f}$, which are all $3-\mathrm{N}$-derivatives. Products $2 \mathbf{a}-\mathbf{f}$ were obtained by standard alkylation on precursors 1a-c [21-23], using appropriately substituted 2-chloro- $\mathrm{N}$-phenylacetamides and $\mathrm{K}_{2} \mathrm{CO}_{3}$ in anhydrous acetonitrile at reflux. The $\mathrm{N}$-aryl derivatives $\mathbf{3 a}-\mathbf{f}$ were obtained through a coupling reaction on $\mathbf{1 a}-\mathbf{c}$ with commercially available phenylboronic acids in the presence of
$\mathrm{EtN}_{3}$ and $(\mathrm{Ac})_{2} \mathrm{Cu}$ (Scheme 1). The reactions presented in Scheme 2 show the formation of both tautomers $(5 \mathbf{a}-\mathbf{c}$ and $\mathbf{6 a - c}$ ), although the $\mathrm{N}$-alkylated derivatives are still the predominant compounds. Lastly, the introduction of a dibenzyl phosphate group on $\mathbf{1 a}-\mathbf{c}$ (Scheme 3) resulted in O-alkylated isomers (10a, b) when using compounds $1 \mathbf{a}$ and $1 \mathbf{c}$, while the same reaction on $\mathbf{1 b}$ led to both isomers 8 and 9 at a 2:1 ratio.

\section{Analytical characterization}

The isomeric compounds 8 and 9 (Scheme 3) feature different positions for the dibenzyl phosphate moiety in their structure. Consequently, a series of MS/MS experiments applying different collision energies (ERMS) were used to characterize these compounds. The breakdown curves obtained from the $[\mathrm{M}+\mathrm{H}]^{+}$ion species of the two isomers are shown in Figs. 3 and 4. Comparison of these fragmentation patterns showed the formation of different product ions between the two isomers. Only the fragment at $406 \mathrm{~m} / \mathrm{z}$ was common for both analytes, but its formation was reached at different collision energies (Figs. 3 and 4). On the basis of these results, the proposed fragmentation patterns of isomers $\mathbf{8}$ and $\mathbf{9}$ are presented in Figs. 5 and 6. As shown in Fig. 5, isomer 8 has two potential fragmentation pathways. The first pathway involves carbonyl sulfide loss, which is favored in heterocyclic moieties, followed by a rearrangement and 

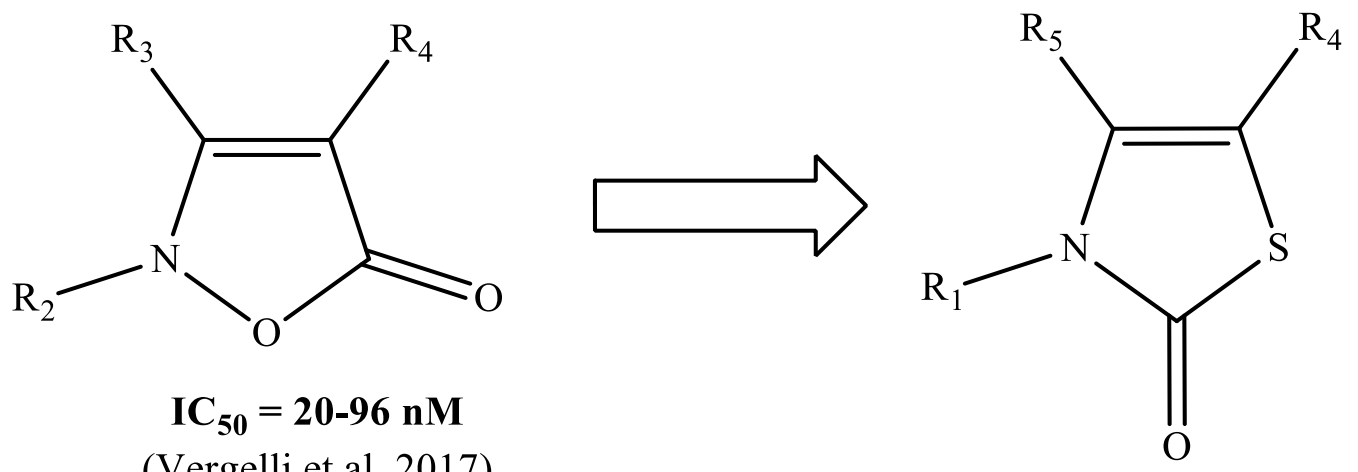

(Vergelli et al. 2017)

Fig. 2 Reference isoxazolone compounds and new thiazolone derivatives

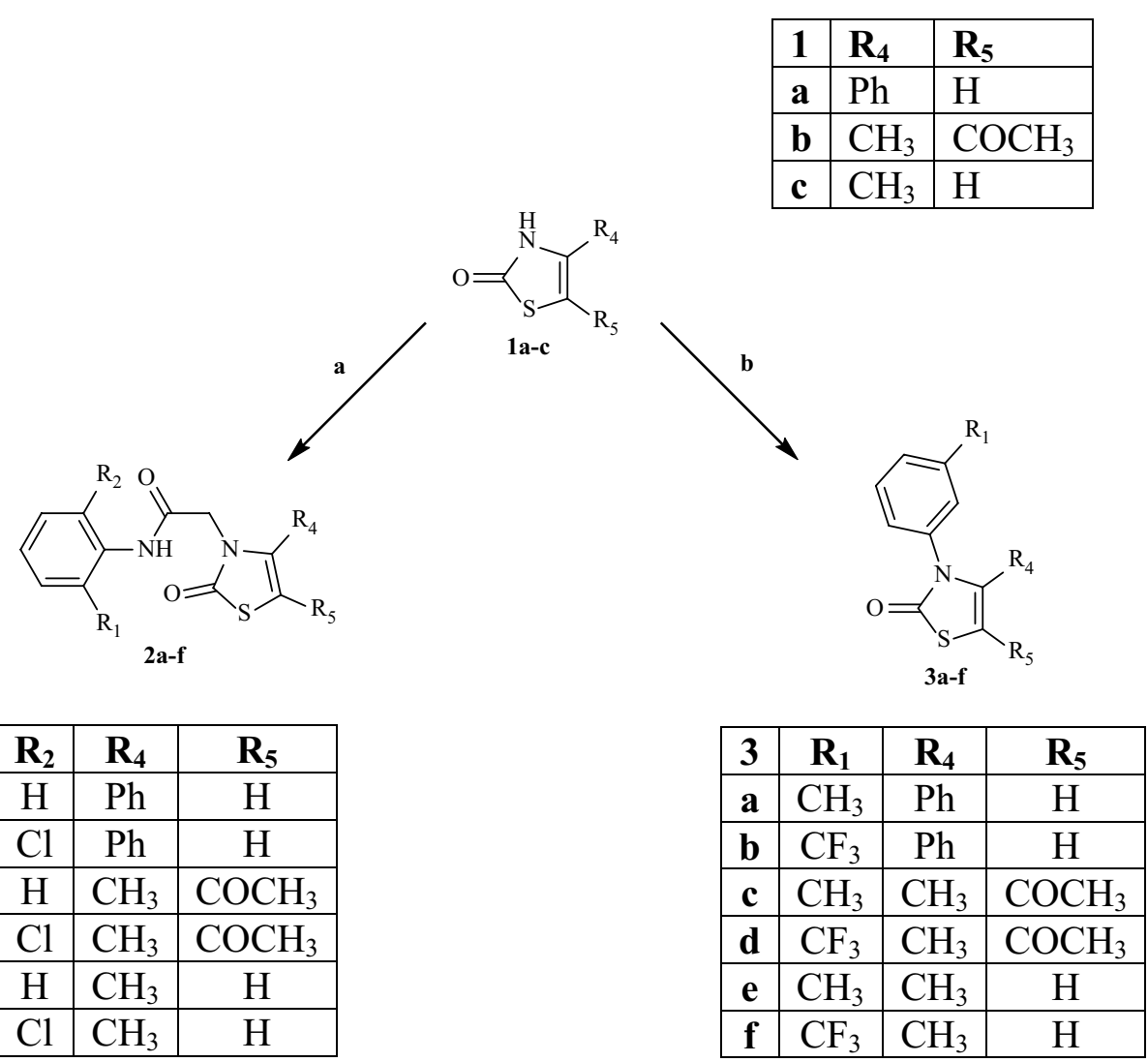

Scheme 1 Reactions and conditions: a appropriate substituted 2-chloro- $N$-phenylacetamide, $\mathrm{CH}_{3} \mathrm{CN}$ anhydrous, $\mathrm{K}_{2} \mathrm{CO}_{3}, 80^{\circ} \mathrm{C}, 6-7 \mathrm{~h} ; \mathbf{b}$ appropriate (substituted)-phenylboronic acid, $\left(\mathrm{CH}_{3} \mathrm{COO}\right)_{2} \mathrm{Cu}, \mathrm{EtN}_{3}, \mathrm{CH}_{2} \mathrm{Cl}_{2}$

loss of benzylphosphate (product ions 388 and $218 \mathrm{~m} / \mathrm{z}$, respectively). The second pathway involves loss of ethenone from the thiazolone ring (product ion $406 \mathrm{~m} / \mathrm{z}$ ).

As shown in Fig. 6, isomer 9 has at least three potential fragmentation ways. The first pathway involves loss of formaldehyde (product ion $418 \mathrm{~m} / \mathrm{z}$ ). The second pathway involves loss of formaldehyde, rearrangement, and loss of benzylphosphate (product ion $248 \mathrm{~m} / \mathrm{z}$ ). The final possible pathway involves loss of ethenone from the thiazolone ring (product ion $406 \mathrm{~m} / \mathrm{z}$ ). Therefore, it is possible that, under MS/MS conditions, isomer 8 represents the $N$-benzylphosphate derivative, leaving the possibility that the carbonyl sulfide could be lost from heterocyclic ring. In contrast, formation of isomer 9 suggests 
<smiles>[R]c1cccc([X])c1</smiles>

\begin{tabular}{|c|c|c|}
\hline $\mathbf{4}$ & $\mathbf{R}_{\mathbf{1}}$ & $\mathbf{X}$ \\
\hline $\mathbf{a}$ & $\mathrm{H}$ & $\mathrm{COO}$ \\
\hline $\mathbf{b}$ & $\mathrm{CH}_{3}$ & - \\
\hline
\end{tabular}

\begin{tabular}{|c|c|c|c|c|}
\hline Comp. & $\mathbf{R}_{\mathbf{1}}$ & $\mathbf{X}$ & $\mathbf{R}_{\mathbf{4}}$ & $\mathbf{R}_{\mathbf{5}}$ \\
\hline $\mathbf{5 a}$ & $\mathrm{H}$ & $\mathrm{COO}$ & $\mathrm{Ph}$ & $\mathrm{H}$ \\
\hline $\mathbf{5 b}$ & $\mathrm{H}$ & $\mathrm{COO}$ & $\mathrm{CH}_{3}$ & $\mathrm{COCH}_{3}$ \\
\hline $\mathbf{5 c}$ & $\mathrm{CH}_{3}$ & - & $\mathrm{Ph}$ & $\mathrm{H}$ \\
\hline $\mathbf{6 a}$ & $\mathrm{H}$ & $\mathrm{COO}$ & $\mathrm{Ph}$ & $\mathrm{H}$ \\
\hline $\mathbf{6 b}$ & $\mathrm{H}$ & $\mathrm{COO}$ & $\mathrm{CH}_{3}$ & $\mathrm{COCH}_{3}$ \\
\hline $\mathbf{6 c}$ & $\mathrm{CH}_{3}$ & - & $\mathrm{Ph}$ & $\mathrm{H}$ \\
\hline
\end{tabular}

Scheme 2 Reactions and conditions: a $\mathrm{CH}_{3} \mathrm{CN}$ anhydrous, $\mathrm{K}_{2} \mathrm{CO}_{3}, 80^{\circ} \mathrm{C}, 3-8 \mathrm{~h}$

that formaldehyde loss is preferred, resulting in occupation of the heterocyclic ring oxygen (O-benzylphosphate isomer).

In order to verify this hypothesis, we extended the MS/MS analysis to other derivatives of the thiazolone ring with the position of the dibenzylphosphate group unknown. MS/MS analysis of the $[\mathrm{M}+\mathrm{H}]^{+}$species of compounds 10a and 10b demonstrated that both analytes lost formaldehyde, with rearrangement of the benzyloxy group (Figs. 7 and 8). This behavior was characteristic of the O-dibenzylphosphate derivative 9 discussed above. Therefore, the structures of compounds 10a and 10b were assigned as O-dibenzylphosphate derivatives.

\section{Results and discussion}

After the correct assignment of the structures, all new compounds were tested as HNE inhibitors (see "Experimental section"). Unfortunately, no activity was found at the highest concentration tested $(40 \mu \mathrm{M})$ for all the compounds in the series. To explain the inability of the investigated compounds to inhibit HNE, we have performed molecular docking studies for docking of compound $\mathbf{2 e}$ into the elastase binding site using MVD software. Our approach was analogous to that with we applied in our earlier investigation of HNE inhibitors [16]. Considering conformational flexibility of the ligand and of the side chains of 42 residues, we found an optimum docking pose of compound 2e into the HNE binding site (Fig. 9). It is known that inhibitory activity towards serine proteases depends on the ability of a compound to form a Michaelis complex with the serine residue at the center of the oxyanion hole and belonging to the catalytic triad Ser...His...Asp [24, 25]. Normally, this complex is formed via nucleophilic attack by the serine oxygen to the carbonyl carbon atom of a ligand and is controlled by geometric peculiarities of the interaction. Namely, the distance $\mathrm{O}($ Ser195)...C(carbonyl in ligand) should be relatively short, and the corresponding angle $\mathrm{O}(\mathrm{Ser} 195) \ldots$ $\mathrm{C}=\mathrm{O}$ should fall within the interval of $80-120$ degrees to support successful formation of the Michaelis complex with HNE $[24,25]$.

We found that compound $\mathbf{2 e}$ is strongly $\mathrm{H}$-bonded to Ser195 by its oxygen atom, and the distance $\mathrm{O}($ Ser195)... $C$ (carbonyl in ligand) is $d_{1}=3.77 \AA$. In addition, the inter-residue distances between heteroatoms in the catalytic triad Ser195...His57...Asp102 were 3.27 and 2.64 A, respectively (Fig. 10). The sum of the latter two distances gives the length of the proton transfer channel $\mathrm{L}=5.91 \AA$. Relatively low values of $\mathrm{d}_{1}$ and $\mathrm{L}$ are favorable for Michaelis complex formation. However, the lowenergy pose of molecule $\mathbf{2 e}$ forms an angle $\mathrm{O}(\mathrm{Ser} 195)$... $\mathrm{C}=\mathrm{O}$ of $12^{\circ}$. This angle is too acute and makes the nucleophilic attack on the carbonyl group impossible with such an orientation of the ligand. It should be noted that the pose is fixed in a narrow binding site cavity (Fig. 9), 


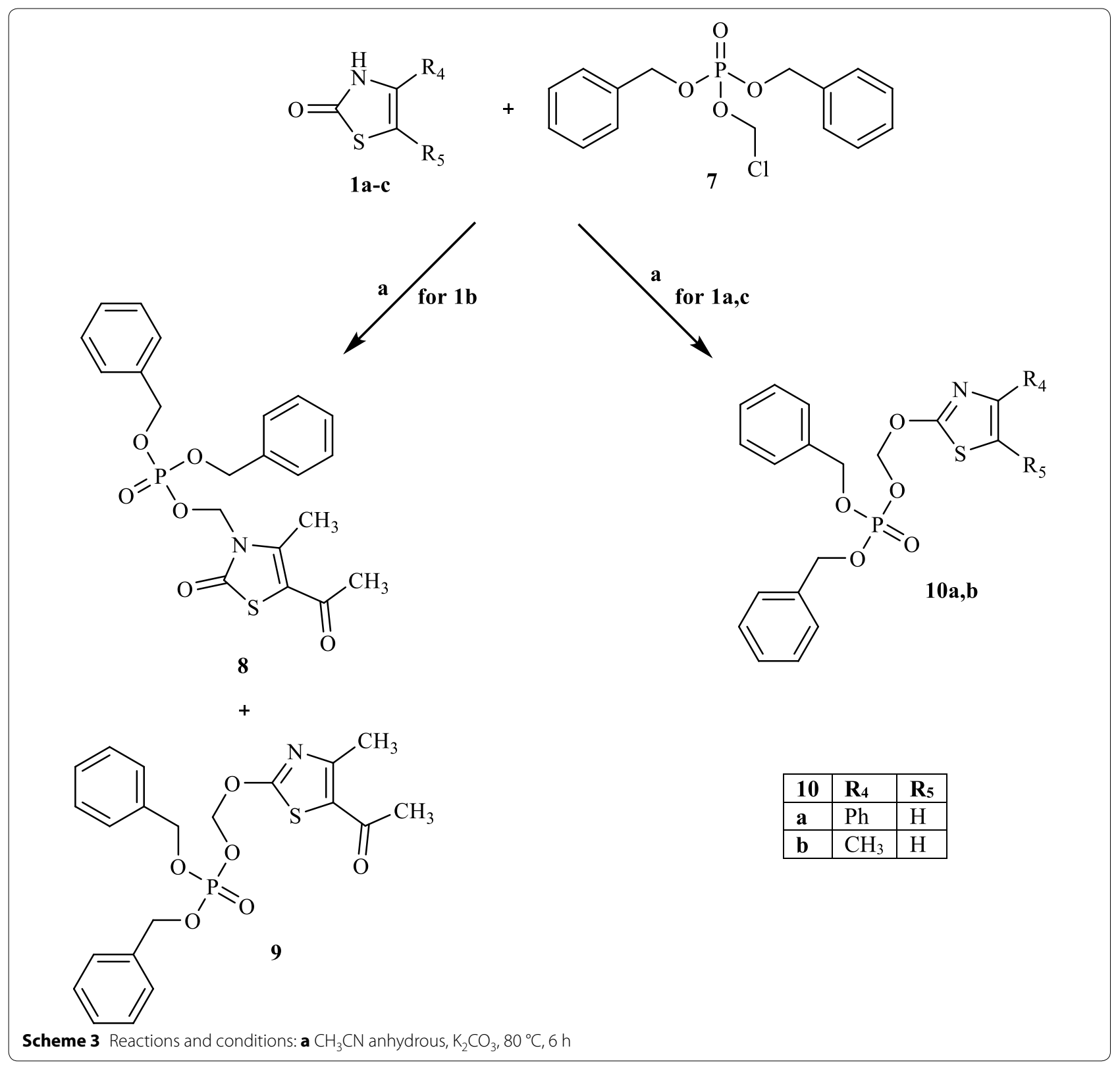

hence the molecule cannot easily change its orientation to satisfy the conditions necessary for the Michaelis complex formation.

\section{Conclusion}

Overall, the thiazol-2-(3H)-one nucleus is not a good scaffold for HNE inhibitors, as the endocyclic carbonyl group does not represent an ideal point of attack for catalytic Ser195 in HNE. Despite the negative result, we obtained some important structure-function information from this study. In general, both the docking and low-energy pose results obtained for compound $\mathbf{2 e}$ explain the lack of inhibitory activity for this library of thiazol-2-(3H)-one-based compounds, as compared to the reference series of isoxazolones [18]. Additionally, compounds $\mathbf{3 a}-\mathbf{f}, \mathbf{5 c}$, and $\mathbf{6 c}$, which have only a carbonyl group at position 2 of the thiazol-2-(3H)-one nucleus, were inactive, confirming the absence of Ser195 nucleophilic attack on the endocyclic carbonyl function of this series. On the other hand, the inactivity of compounds 2a-f, 5a, b, and $\mathbf{6 a}, \mathbf{b}$, bearing a different carbonyl group (i.e., amide or ester) at $\mathrm{N}-1$, suggests that the thiazol2-(3H)-one nucleus itself is not a suitable scaffold for HNE inhibitors. The lack of activity for compounds 8-10 


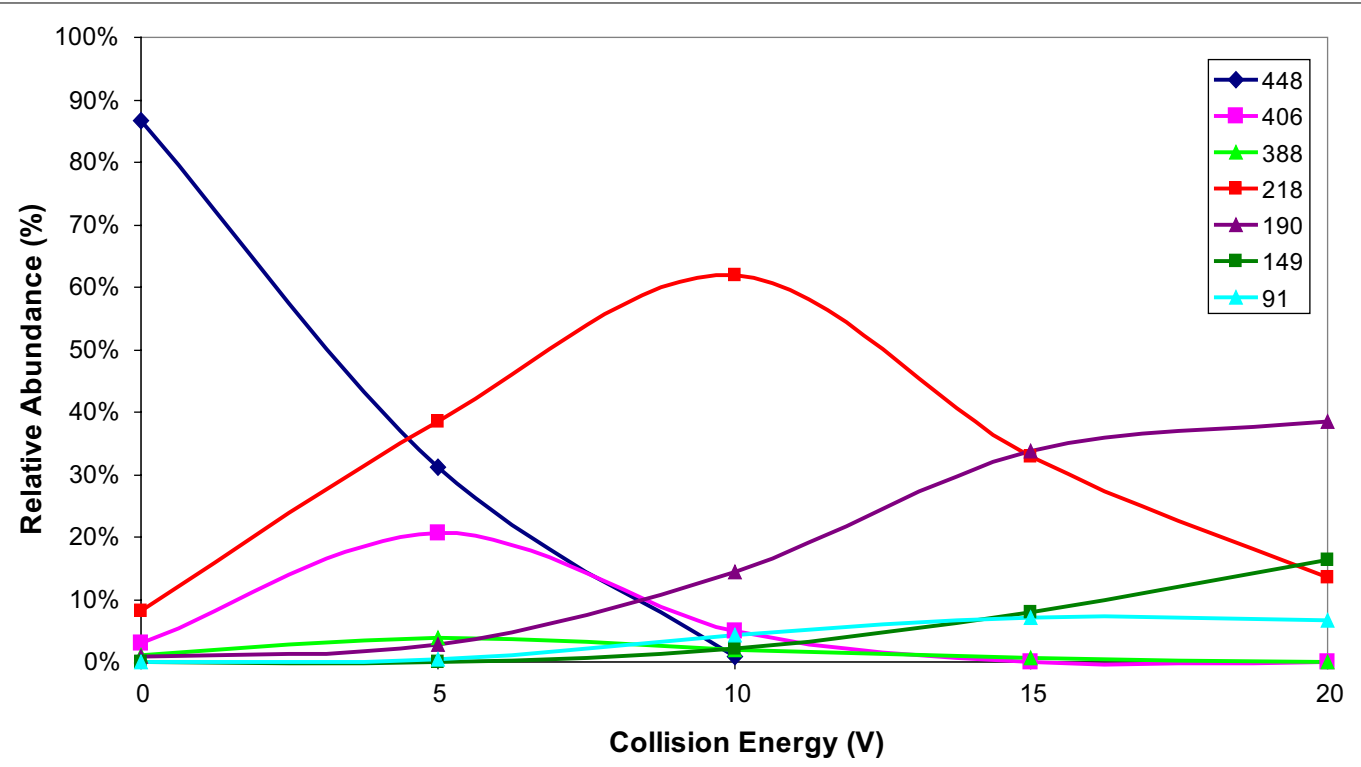

Fig. 3 Breakdown curves obtained from ERMS experiment on the compound 8

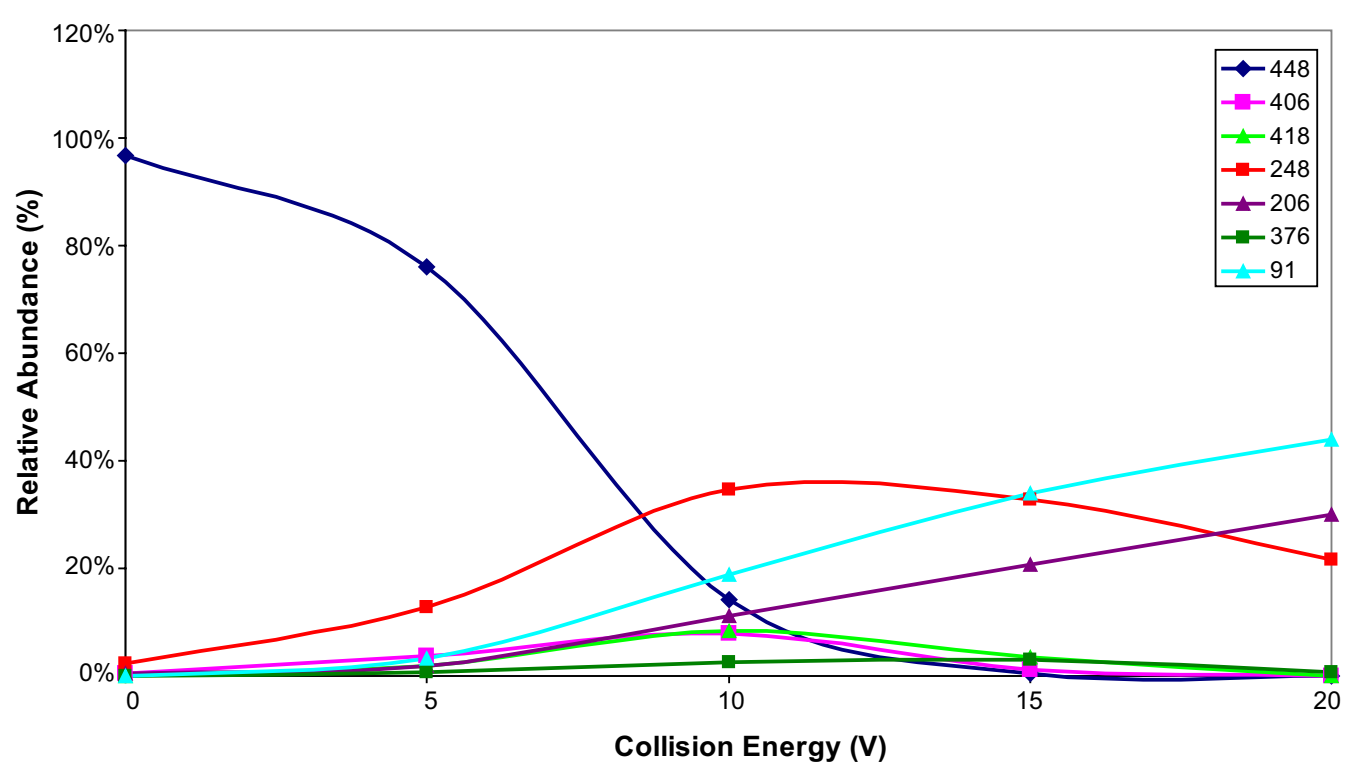

Fig. 4 Breakdown curves obtained from ERMS experiment on the compound 9

is a further support for this conclusion, considering the presence of a bulky phosphonic fragment, which is a typical residue in potent HNE inhibitors reported in the literature [26].

\section{Experimental section}

\section{Chemistry}

All melting points were determined on a Büchi apparatus and are uncorrected. ${ }^{1} \mathrm{H}$ NMR, ${ }^{13} \mathrm{C}$-NMR, HSQC and
HMBC spectra were recorded on an Avance 400 instrument (Bruker Biospin Version 002 with SGU). Chemical shifts are reported in $\mathrm{ppm}$, using the solvent as internal standard. Extracts were dried over $\mathrm{Na}_{2} \mathrm{SO}_{4}$, and the solvents were removed under reduced pressure. Merck F-254 commercial plates were used for analytical TLC to follow the course of the reactions. Silica gel 60 (Merck 70-230 mesh) was used for column chromatography. Microanalyses were performed with a Perkin-Elmer 260 


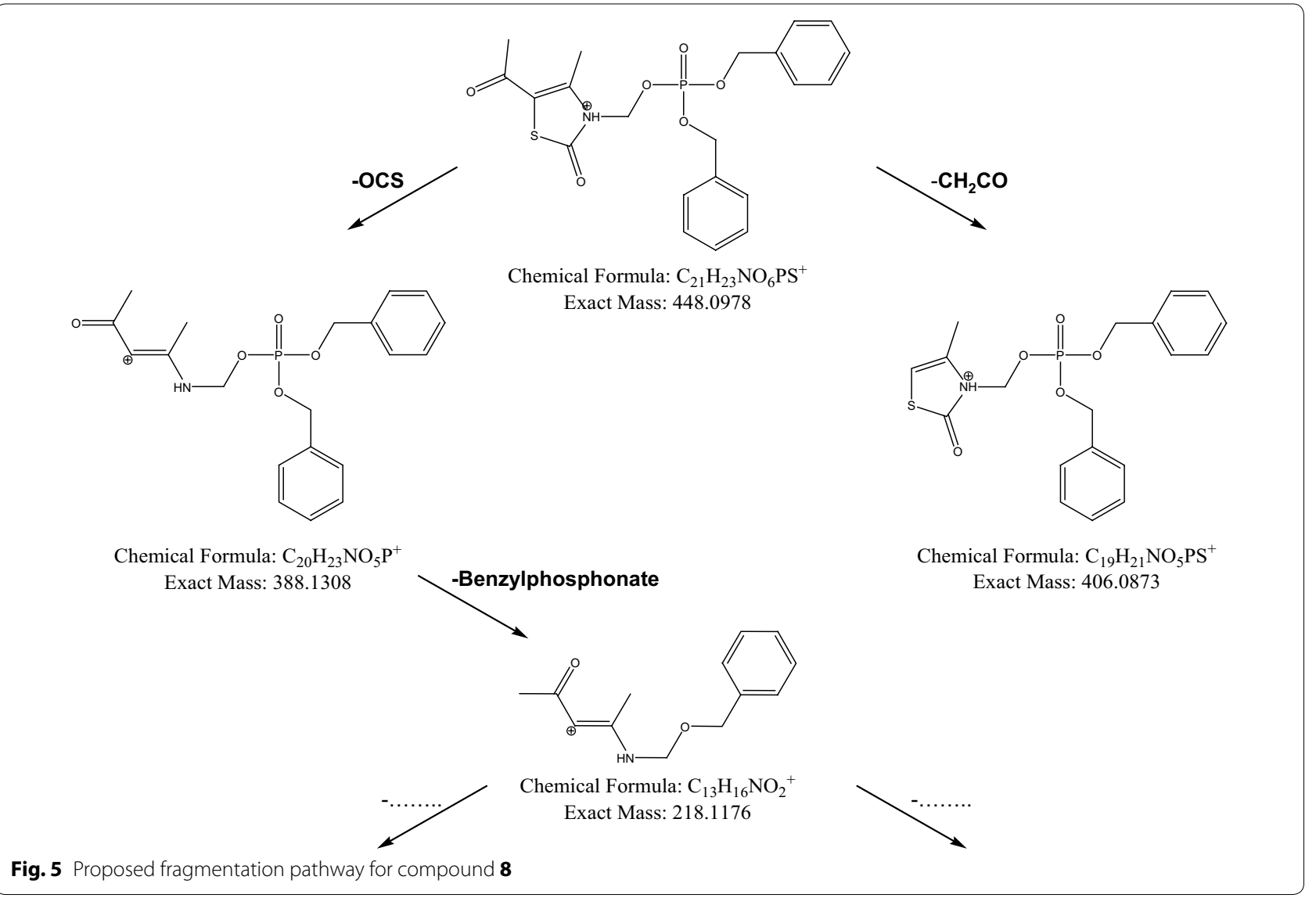

elemental analyzer for $\mathrm{C}, \mathrm{H}$, and $\mathrm{N}$, and the results were within $\pm 0.4 \%$ of the theoretical values unless otherwise stated. Reagents and starting material were commercially available.

\section{General procedure for $2 a-f$}

$1.12 \mathrm{mmol}$ of $\mathrm{K}_{2} \mathrm{CO}_{3}$ and $0.67 \mathrm{mmol}$ (for $2 \mathbf{a}-\mathbf{f}$ ) of the appropriate 2-chloro- $N$-phenylacetamide were added to a suspension of intermediate 1a-c [21-23] $(0.56 \mathrm{mmol})$ in anhydrous $\mathrm{CH}_{3} \mathrm{CN}(3 \mathrm{~mL})$. The mixture was stirred at reflux for 6-7 h. After evaporation of the solvent, ice-cold water $(20 \mathrm{~mL})$ was added, and the precipitate was recovered by vacuum filtration. Final compounds $2 \mathbf{a}-\mathbf{f}$ were purified by column chromatography using cyclohexane/ethyl acetate 2:1 (2a, b, e), 1:2 (2c, d) and 1:1 (2f) as eluents.

\section{2-(2-Oxo-4-phenylthiazol-3(2H)-yl)-N-phenylacetamide (2a)}

Yield $=34 \% ; \mathrm{mp}=177-179{ }^{\circ} \mathrm{C}(\mathrm{EtOH}) \cdot{ }^{1} \mathrm{H}-\mathrm{NMR}$ $\left(\mathrm{CDCl}_{3}\right) \delta 4.45\left(\mathrm{~s}, 2 \mathrm{H}, \mathrm{CH}_{2}\right), 6.14(\mathrm{~s}, 1 \mathrm{H}, \mathrm{CH}), 7.07$ $(\mathrm{t}, 1 \mathrm{H}, \mathrm{Ar}, J=7.2 \mathrm{~Hz}), 7.25(\mathrm{t}, 2 \mathrm{H}, \mathrm{Ar}, J=7.6 \mathrm{~Hz})$,
7.40-7.50 (m, 7H, Ar), 8.63 (exch br s, $1 \mathrm{H}, \mathrm{NH}) .{ }^{13} \mathrm{C}-$ NMR $(\mathrm{CDCl} 3) \delta 53.40\left(\mathrm{CH}_{2}\right), 110.53(\mathrm{CH}), 121.61$ $(\mathrm{CH}), 121.65(\mathrm{CH}), 127.90(\mathrm{CH}), 128.05(\mathrm{CH}), 128.32$ $(\mathrm{CH}), 128.36(\mathrm{CH}), 128.63(\mathrm{CH}), 128.68(\mathrm{CH}), 128.91$ $(\mathrm{CH}), 128.96(\mathrm{CH}), 130.70(\mathrm{C}), 131.92(\mathrm{C}), 138.50$ (C), 168.54 (C), 169.93 (C). IR: $1599 \mathrm{~cm}^{-1}(\mathrm{C}=\mathrm{O})$, $1659 \mathrm{~cm}^{-1}(\mathrm{C}=\mathrm{O}), 3262 \mathrm{~cm}^{-1}(\mathrm{NH})$. LC-MS: 311.0 $[\mathrm{M}+\mathrm{H}]^{+}$.

\section{$\mathrm{N}$-(2,6-dichlorophenyl)-2-(2-oxo-4-phenylthiazol-3(2H)-yl)} acetamide (2b)

Yield $=10 \% ; \mathrm{mp}=213-216{ }^{\circ} \mathrm{C}(\mathrm{EtOH}) .{ }^{1} \mathrm{H}-\mathrm{NMR}$ $\left(\mathrm{CDCl}_{3}\right) \delta 4.51\left(\mathrm{~s}, 2 \mathrm{H}, \mathrm{CH}_{2}\right), 6.18(\mathrm{~s}, 1 \mathrm{H}, \mathrm{CH}), 7.21(\mathrm{t}, 1 \mathrm{H}$, Ar, $J=8.0 \mathrm{~Hz}$ ), 7.39 (d, 2H, Ar, $J=8.4 \mathrm{~Hz}), 7.45-7.55$ (m, 5H, Ar), 8.22 (exch br s, $1 \mathrm{H}, \mathrm{NH}) .{ }^{13} \mathrm{C}-\mathrm{NMR}(\mathrm{CDCl} 3)$ $\delta 53.41\left(\mathrm{CH}_{2}\right), 110.50(\mathrm{CH}), 126.11(\mathrm{CH}), 127.90(\mathrm{CH})$, $128.24(\mathrm{CH}), 128.27(\mathrm{CH}), 128.33(\mathrm{CH}), 128.36(\mathrm{CH})$, $128.63(\mathrm{CH}), 128.69(\mathrm{CH}), 130.70(\mathrm{C}), 131.94(\mathrm{C}), 132.06$ (C), 133.30 (C), 133.35 (C) 168.50 (C), 169.96 (C). IR: $1585 \mathrm{~cm}^{-1}(\mathrm{C}=\mathrm{O}), 1646 \mathrm{~cm}^{-1}(\mathrm{C}=\mathrm{O}), 3205 \mathrm{~cm}^{-1}(\mathrm{NH})$. LC-MS: $379.9[\mathrm{M}+\mathrm{H}]^{+}$. 

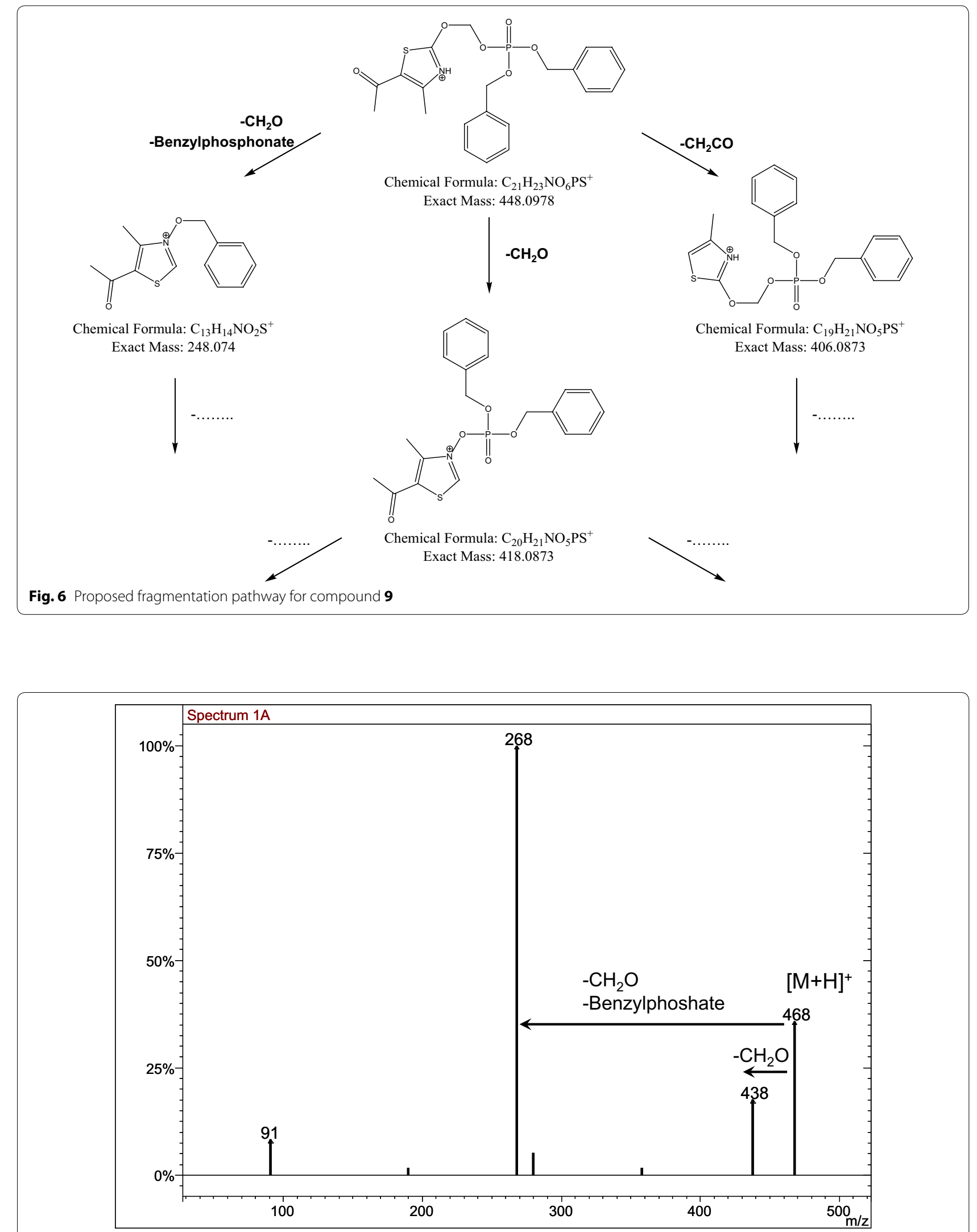

Fig. $7 \mathrm{MS} / \mathrm{MS}$ spectra of $[\mathrm{M}+\mathrm{H}]^{+}$species of compound $\mathbf{1 0 a}$ 
Crocetti et al. Chemistry Central Journal (2017) 11:127

Page 9 of 15

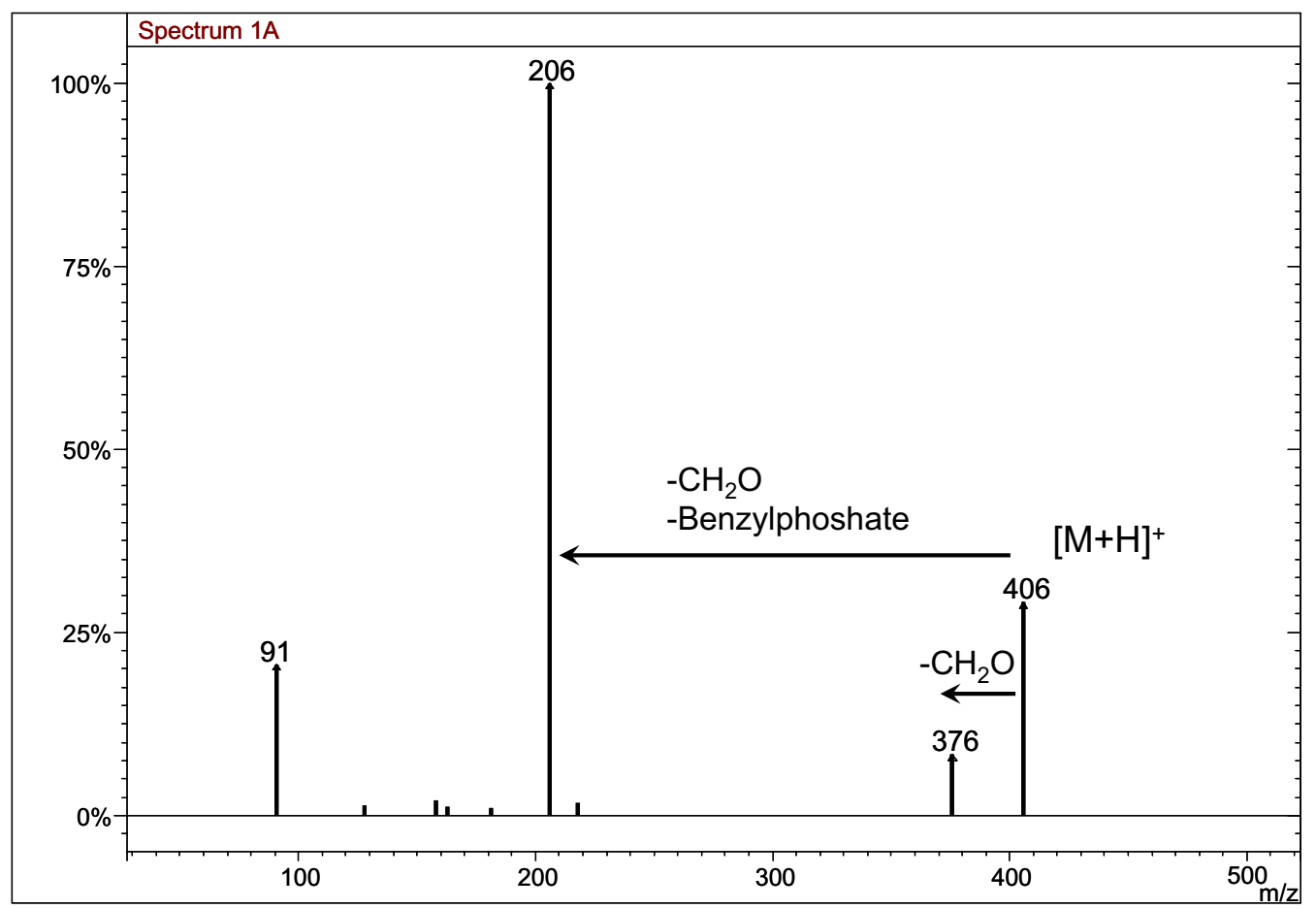

Fig. $8 \mathrm{MS} / \mathrm{MS}$ spectra of $[\mathrm{M}+\mathrm{H}]^{+}$species of compound $\mathbf{1 0 b}$

Fig. 9 Docking pose of compound $\mathbf{2 e}$ within the HNE ligand-binding site 


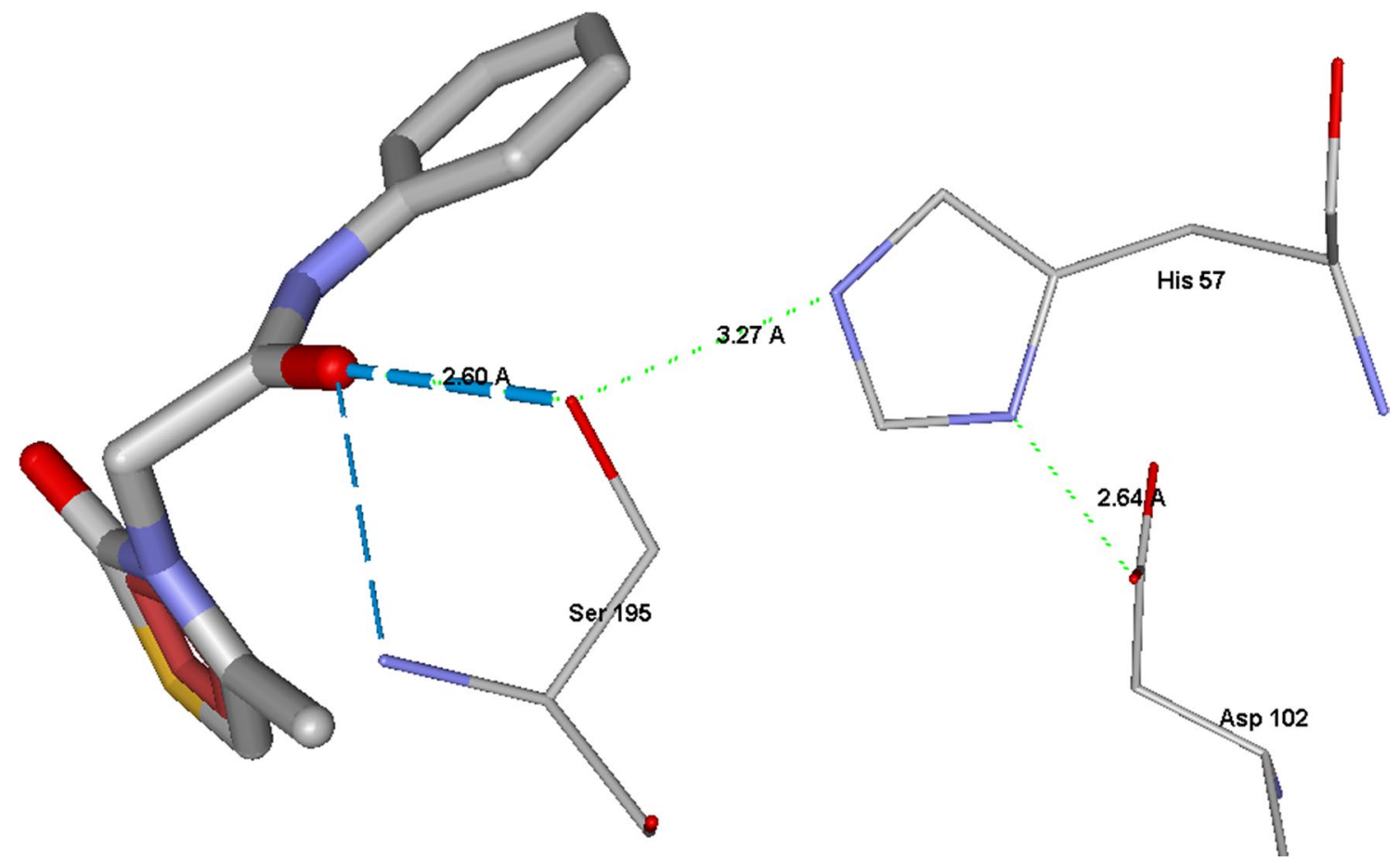

Fig. 10 Orientation compound $\mathbf{2 e}$ docking pose (thick cylinders) with respect to residues of the catalytic triad (thin cylinders). Hydrogen atoms are hidden for clarity. Hydrogen bonds are shown by blue dashed lines. Segments of the proton transfer channel are indicated as light-green dashed lines

2-(5-Acetyl-4-methyl-2-oxothiazol-3(2H)-yl)-N-phenylacetamide (2c)

Yield $=16 \% ; \mathrm{mp}=188-190{ }^{\circ} \mathrm{C}(\mathrm{EtOH}) .{ }^{1} \mathrm{H}-\mathrm{NMR}$ $\left(\mathrm{CDCl}_{3}\right) \delta 2.40\left(\mathrm{~s}, 3 \mathrm{H}, \mathrm{CH}_{3}\right), 2.68\left(\mathrm{~s}, 3 \mathrm{H}, \mathrm{COCH}_{3}\right), 4.59$ $\left(\mathrm{s}, 2 \mathrm{H}, \mathrm{CH}_{2}\right), 7.15(\mathrm{t}, 1 \mathrm{H}, \mathrm{Ar}, J=7.4 \mathrm{~Hz}), 7.33(\mathrm{t}, 2 \mathrm{H}, \mathrm{Ar}$, $J=7.6 \mathrm{~Hz}$ ), 7.49 (d, 2H, Ar, $J=8.0 \mathrm{~Hz}$ ), 8.10 (exch br s, $1 \mathrm{H}, \mathrm{NH}) .{ }^{13} \mathrm{C}-\mathrm{NMR}\left(\mathrm{CDCl}_{3}\right) \delta 14.70\left(\mathrm{CH}_{3}\right), 26.14\left(\mathrm{CH}_{3}\right)$, $53.52\left(\mathrm{CH}_{2}\right), 107.41(\mathrm{C}), 121.60(\mathrm{CH}), 121.66(\mathrm{CH}), 128.04$ $(\mathrm{CH}), 128.92(\mathrm{CH}), 128.97(\mathrm{CH}), 138.55(\mathrm{C}), 145.68(\mathrm{C})$, 168.53 (C), 169.91 (C), 192.70 (C). IR: $1575 \mathrm{~cm}^{-1}(\mathrm{C}=\mathrm{O})$, $1643 \mathrm{~cm}^{-1}(\mathrm{C}=\mathrm{O}), 1698 \mathrm{~cm}^{-1}(\mathrm{C}=\mathrm{O}), 3295 \mathrm{~cm}^{-1}(\mathrm{NH})$. LC-MS: $291.1[\mathrm{M}+\mathrm{H}]^{+}$.

\section{2-(5-Acetyl-4-methyl-2-oxothiazol-3(2H)-yl)-N-(2,6-dichloro-} phenyl)acetamide (2d)

Yield $=15 \% ; \mathrm{mp}=209-211{ }^{\circ} \mathrm{C}(\mathrm{EtOH}) .{ }^{1} \mathrm{H}-\mathrm{NMR}$ $\left(\mathrm{CDCl}_{3}\right) \delta 2.40\left(\mathrm{~s}, 3 \mathrm{H}, \mathrm{CH}_{3}\right), 2.68\left(\mathrm{~s}, 3 \mathrm{H}, \mathrm{COCH}_{3}\right)$, $4.69\left(\mathrm{~s}, 2 \mathrm{H}, \mathrm{CH}_{2}\right), 7.21(\mathrm{t}, 1 \mathrm{H}, \mathrm{Ar}, J=8.0 \mathrm{~Hz}), 7.37(\mathrm{~d}$, $2 \mathrm{H}, \mathrm{Ar}, J=8.4 \mathrm{~Hz}$ ), 7.82 (exch br s, $1 \mathrm{H}, \mathrm{NH}) .{ }^{13} \mathrm{C}-\mathrm{NMR}$ $(\mathrm{CDCl} 3) \delta 14.74\left(\mathrm{CH}_{3}\right), 26.11\left(\mathrm{CH}_{3}\right), 53.56\left(\mathrm{CH}_{2}\right), 107.40$ (C), $126.13(\mathrm{CH}), 128.22(\mathrm{CH}), 128.26(\mathrm{CH}), 132.05(\mathrm{C})$, 133.30 (C), 133.33 (C), 145.68 (C), 168.52 (C), 169.93 (C), 192.71 (C). IR: $1573 \mathrm{~cm}^{-1}(\mathrm{C}=\mathrm{O}) ; 1637 \mathrm{~cm}^{-1}$ $(\mathrm{C}=\mathrm{O}), 1672 \mathrm{~cm}^{-1}(\mathrm{C}=\mathrm{O}), 3202 \mathrm{~cm}^{-1}(\mathrm{NH})$. LC-MS: $359.9[\mathrm{M}+\mathrm{H}]^{+}$.
2-(4-Methyl-2-oxothiazol-3(2H)-yl)-N-phenylacetamide (2e) Yield $=26 \% ; \mathrm{mp}=188-191{ }^{\circ} \mathrm{C}(\mathrm{EtOH}) .{ }^{1} \mathrm{H}-\mathrm{NMR}$ $\left(\mathrm{CDCl}_{3}\right) \delta 2.26\left(\mathrm{~s}, 3 \mathrm{H}, \mathrm{CH}_{3}\right), 4.51\left(\mathrm{~s}, 2 \mathrm{H}, \mathrm{CH}_{2}\right), 5.86(\mathrm{~s}$, $1 \mathrm{H}, \mathrm{CH}), 7.13(\mathrm{t}, 1 \mathrm{H}, \mathrm{Ar}, J=7.4 \mathrm{~Hz}), 7.32(\mathrm{t}, 2 \mathrm{H}, \mathrm{Ar}$, $J=7.6 \mathrm{~Hz}$ ), 7.50 (d, 2H, Ar, $J=8.0 \mathrm{~Hz}$ ), 8.39 (exch br s, $1 \mathrm{H}, \mathrm{NH}) .{ }^{13} \mathrm{C}$-NMR $(\mathrm{CDCl} 3) \delta 17.32\left(\mathrm{CH}_{3}\right), 53.51\left(\mathrm{CH}_{2}\right)$, $101.90(\mathrm{CH}), 120.45(\mathrm{C}), 121.63(\mathrm{CH}), 121.67(\mathrm{CH})$, 128.04 (CH), $128.93(\mathrm{CH}), 128.98(\mathrm{CH}), 138.50(\mathrm{C})$, $168.58(\mathrm{C}), 169.92(\mathrm{C})$. IR: $1633 \mathrm{~cm}^{-1}(\mathrm{C}=\mathrm{O}), 1692 \mathrm{~cm}^{-1}$ $(\mathrm{C}=\mathrm{O}), 3274 \mathrm{~cm}^{-1}(\mathrm{NH})$. LC-MS: $249.1[\mathrm{M}+\mathrm{H}]^{+}$.

\section{$\mathrm{N}$-(2,6-dichlorophenyl)-2-(4-methyl-2-oxothiazol-3(2H)-yl)} acetamide (2f)

Yield $=9 \% ; \mathrm{mp}=266-269{ }^{\circ} \mathrm{C}(\mathrm{EtOH}) .{ }^{1} \mathrm{H}-\mathrm{NMR}\left(\mathrm{CDCl}_{3}\right) \delta$ $2.28\left(\mathrm{~s}, 3 \mathrm{H}, \mathrm{CH}_{3}\right), 4.61$ (s, $\left.2 \mathrm{H}, \mathrm{CH}_{2}\right), 5.89$ (s, $\left.1 \mathrm{H}, \mathrm{CH}\right), 7.20$ (t, $1 \mathrm{H}, \mathrm{Ar}, J=8.0 \mathrm{~Hz}$ ), 7.37 (d, 2H, Ar, $J=8.0 \mathrm{~Hz}$ ), 7.90 (exch br s, $1 \mathrm{H}, \mathrm{NH}) .{ }^{13} \mathrm{C}-\mathrm{NMR}(\mathrm{CDCl} 3) \delta 17.30\left(\mathrm{CH}_{3}\right)$, $53.52\left(\mathrm{CH}_{2}\right), 101.90(\mathrm{CH}), 120.48(\mathrm{C}), 126.11(\mathrm{CH}), 128.24$ $(\mathrm{CH}), 128.29(\mathrm{CH}), 132.01(\mathrm{C}), 133.33(\mathrm{C}), 133.38(\mathrm{C})$, 168.50 (C), $169.91(\mathrm{C})$. IR: $1634 \mathrm{~cm}^{-1}(\mathrm{C}=\mathrm{O}), 1685 \mathrm{~cm}^{-1}$ $(\mathrm{C}=\mathrm{O}), 3197 \mathrm{~cm}^{-1}(\mathrm{NH}) . \mathrm{LC}-\mathrm{MS}: 317.9[\mathrm{M}+\mathrm{H}]^{+}$.

\section{General procedure for $3 a-f$}

A mixture of intermediate of type $\mathbf{1}(\mathbf{1 a}-\mathbf{c})$ [21-23] $(0.62 \mathrm{mmol})$ the appropriate phenylboronic acid 
(1.24 mmol), $\mathrm{Cu}(\mathrm{Ac})_{2}(0.93 \mathrm{~m} \mathrm{~mol})$, and $\mathrm{Et}_{3} \mathrm{~N}(1.24 \mathrm{mmol})$ in dry $\mathrm{CH}_{2} \mathrm{Cl}_{2}(3 \mathrm{~mL})$, was stirred at room temperature overnight. The organic layer was washed with water $(3 \times 15 \mathrm{~mL})$ and then with $33 \%$ aqueous ammonia $(3 \times 10 \mathrm{~mL})$. The organic layer was dried over sodium sulfate and the solvent was evaporated in vacuo to obtain final compounds $\mathbf{3 a}-\mathbf{f}$, which were purified by column chromatography using cyclohexane/ethyl acetate 5:1 (for 3a), 2:1 (for $\mathbf{3 b}$ ) or 3:1 (for $\mathbf{3 c - f}$ ) as eluents.

\section{4-Phenyl-3-m-tolyl-thiazol-2(3H)-one (3a)}

Yield $=12 \%$; $\mathrm{mp}=118-121{ }^{\circ} \mathrm{C}(\mathrm{EtOH}) .{ }^{1} \mathrm{H}-\mathrm{NMR}$ $\left(\mathrm{CDCl}_{3}\right) \delta 2.31\left(\mathrm{~s}, 3 \mathrm{H}, \mathrm{CH}_{3}\right), 6.20(\mathrm{~s}, 1 \mathrm{H}, \mathrm{CH}), 6.88(\mathrm{~d}, 1 \mathrm{H}$, $\mathrm{Ar}, J=7.6 \mathrm{~Hz}$ ), 7.02 (s, 1H, Ar), 7.09-7.15 (m, 2H, Ar), 7.18-7.26 (m, 5H, Ar). ${ }^{13} \mathrm{C}-\mathrm{NMR}(\mathrm{CDCl} 3) \delta 21.30\left(\mathrm{CH}_{3}\right)$, $106.33(\mathrm{CH}), 124.67(\mathrm{CH}), 125.11(\mathrm{CH}), 127.92(\mathrm{CH})$, $128.30(\mathrm{CH}), 128.35(\mathrm{CH}), 128.63(\mathrm{CH}), 128.68(\mathrm{CH})$, $128.88(\mathrm{CH}), 129.55(\mathrm{CH}), 130.41(\mathrm{C}), 132.64(\mathrm{C}), 138.62$ (C), 147.36 (C), 166.70 (C). IR: $1658 \mathrm{~cm}^{-1}$ (C=O). LCMS: $268.1[\mathrm{M}+\mathrm{H}]^{+}$.

4-Phenyl-3-[3-(trifluoromethyl)phenyl]thiazol-2(3H)-one (3b) Yield $=15 \% ; \mathrm{mp}=84-87{ }^{\circ} \mathrm{C}(\mathrm{EtOH}) .{ }^{1} \mathrm{H}-\mathrm{NMR}\left(\mathrm{CDCl}_{3}\right)$ $\delta 6.26(\mathrm{~s}, 1 \mathrm{H}, \mathrm{CH}), 7.06(\mathrm{~d}, 2 \mathrm{H}, \mathrm{Ar}, J=7.6 \mathrm{~Hz}), 7.24-$ $7.30(\mathrm{~m}, 3 \mathrm{H}, \mathrm{Ar}), 7.35-7.40(\mathrm{~m}, 2 \mathrm{H}, \mathrm{Ar}), 7.47(\mathrm{t}, 1 \mathrm{H}$, Ar, $J=7.8 \mathrm{~Hz}), 7.55$ (d, $1 \mathrm{H}, \mathrm{Ar}, J=7.6 \mathrm{~Hz}) \cdot{ }^{13} \mathrm{C}-\mathrm{NMR}$ (CDCl3) $\delta 106.30(\mathrm{CH}), 120.72(\mathrm{CH}), 124.11(\mathrm{C}), 125.93$ $(\mathrm{CH}), 127.97(\mathrm{CH}), 128.35(\mathrm{CH}), 128.39(\mathrm{CH}), 128.65$ $(\mathrm{CH}), 128.68(\mathrm{CH}), 129.25(\mathrm{CH}), 130.41(\mathrm{C}), 131.24$ (C), $131.40(\mathrm{CH}), 133.06(\mathrm{C}), 147.35(\mathrm{C}), 166.71(\mathrm{C}) . \mathrm{IR}$ : $1656 \mathrm{~cm}^{-1}(\mathrm{C}=\mathrm{O})$. LC-MS: $322.0[\mathrm{M}+\mathrm{H}]^{+}$.

\section{5-Acetyl-4-methyl-3-m-tolylthiazol-2(3H)-one (3C)}

Yield $=12 \% ; \mathrm{mp}=104-106{ }^{\circ} \mathrm{C}(\mathrm{EtOH}) .{ }^{1} \mathrm{H}-\mathrm{NMR}$ $\left(\mathrm{CDCl}_{3}\right) \delta 2.31\left(\mathrm{~s}, 3 \mathrm{H}, \mathrm{CH}_{3}\right), 2.42\left(\mathrm{~s}, 3 \mathrm{H}, \mathrm{COCH}_{3}\right), 2.43$ (s, 3H, $\left.\mathrm{CH}_{3}-\mathrm{Ph}\right), 7.04-7.09(\mathrm{~m}, 2 \mathrm{H}, \mathrm{Ar}), 7.32(\mathrm{~d}, 1 \mathrm{H}$, $\mathrm{Ar}, J=7.6 \mathrm{~Hz}), 7.42(\mathrm{t}, 1 \mathrm{H}, \mathrm{Ar}, \mathrm{J}=7.6 \mathrm{~Hz}) \cdot{ }^{13} \mathrm{C}-\mathrm{NMR}$ $(\mathrm{CDCl} 3) \delta 15.03\left(\mathrm{CH}_{3}\right), 21.33\left(\mathrm{CH}_{3}\right), 26.12\left(\mathrm{CH}_{3}\right), 103.27$ (C), $124.60(\mathrm{CH}), 125.14(\mathrm{CH}), 128.88(\mathrm{CH}), 129.55(\mathrm{CH})$, $132.60(\mathrm{C}), 138.62(\mathrm{C}), 154.03(\mathrm{C}), 166.74(\mathrm{C}), 192.71(\mathrm{C})$. IR: $1629 \mathrm{~cm}^{-1}(\mathrm{C}=\mathrm{O}), 1670 \mathrm{~cm}^{-1}(\mathrm{C}=\mathrm{O})$. LC-MS: 248.1 $[\mathrm{M}+\mathrm{H}]^{+}$.

\section{5-Acetyl-4-methyl-3-[3-(trifluoromethyl)phenyl]thia-}

zol-2(3H)-one (3d)

Yield $=11 \% ; \mathrm{mp}=147-150{ }^{\circ} \mathrm{C}(\mathrm{EtOH}) .{ }^{1} \mathrm{H}-\mathrm{NMR}$ $\left(\mathrm{CDCl}_{3}\right) \delta 2.33\left(\mathrm{~s}, 3 \mathrm{H}, \mathrm{CH}_{3}\right), 2.43\left(\mathrm{~s}, 3 \mathrm{H}, \mathrm{COCH}_{3}\right), 7.48$ (d, $1 \mathrm{H}, \mathrm{Ar}, J=8.0 \mathrm{~Hz}), 7.53(\mathrm{~s}, 1 \mathrm{H}, \mathrm{Ar}), 7.71(\mathrm{t}, 1 \mathrm{H}$, Ar, $J=7.8 \mathrm{~Hz}), 7.80(\mathrm{~d}, 1 \mathrm{H}, \mathrm{Ar}, \mathrm{J}=8.0 \mathrm{~Hz}) \cdot{ }^{13} \mathrm{C}-\mathrm{NMR}$ $(\mathrm{CDCl} 3) \delta 15.05\left(\mathrm{CH}_{3}\right), 26.14\left(\mathrm{CH}_{3}\right), 103.25(\mathrm{C}), 120.70$ $(\mathrm{CH}), 124.13(\mathrm{C}), 125.97(\mathrm{CH}), 129.24(\mathrm{CH}), 131.22(\mathrm{C})$, 131.40 (CH), 133.04 (C), 154.08 (C), 166.77 (C), 192.75
(C). IR: $1637 \mathrm{~cm}^{-1}(\mathrm{C}=\mathrm{O}), 1681 \mathrm{~cm}^{-1}(\mathrm{C}=\mathrm{O})$. LC-MS: $302.0[\mathrm{M}+\mathrm{H}]^{+}$.

4-Methyl-3-m-tolyl-thiazol-2(3H)-one (3e)

Yield $=11 \% ; \mathrm{mp}=69-72{ }^{\circ} \mathrm{C}(\mathrm{EtOH}) .{ }^{1} \mathrm{H}-\mathrm{NMR}\left(\mathrm{CDCl}_{3}\right)$ $\delta 1.90\left(\mathrm{~s}, 3 \mathrm{H}, \mathrm{CH}_{3}\right), 2.42\left(\mathrm{~s}, 3 \mathrm{H}, \mathrm{CH}_{3}-\mathrm{Ph}\right), 5.86(\mathrm{~s}, 1 \mathrm{H}$, $\mathrm{CH}), 7.05-7.10(\mathrm{~m}, 2 \mathrm{H}, \mathrm{Ar}), 7.25(\mathrm{~s}, 1 \mathrm{H}, \mathrm{Ar}), 7.39(\mathrm{t}$, $1 \mathrm{H}, \mathrm{Ar}, J=7.6 \mathrm{~Hz}) .{ }^{13} \mathrm{C}-\mathrm{NMR}(\mathrm{CDCl} 3) \delta 21.32\left(\mathrm{CH}_{3}\right)$, $21.79\left(\mathrm{CH}_{3}\right), 97.70(\mathrm{CH}), 124.65(\mathrm{CH}), 125.16(\mathrm{CH})$, $128.83(\mathrm{CH}), 129.51(\mathrm{CH}), 132.64(\mathrm{C}), 138.60(\mathrm{C}), 149.33$ (C), $166.75(\mathrm{C})$. IR: $1640 \mathrm{~cm}^{-1}(\mathrm{C}=\mathrm{O})$. LC-MS: 206.0 $[\mathrm{M}+\mathrm{H}]^{+}$.

4-Methyl-3-[3-(trifluoromethyl)phenyl]thiazol-2(3H)-one (3f) Yield = 18\%; Oil. ${ }^{1} \mathrm{H}-\mathrm{NMR}\left(\mathrm{CDCl}_{3}\right) \delta 1.92\left(\mathrm{~s}, 3 \mathrm{H}, \mathrm{CH}_{3}\right)$, $5.92(\mathrm{~s}, 1 \mathrm{H}, \mathrm{CH}), 7.50(\mathrm{~d}, 1 \mathrm{H}, \mathrm{Ar}, J=7.6 \mathrm{~Hz}), 7.56(\mathrm{~s}$, $1 \mathrm{H}, \mathrm{Ar}), 7.66(\mathrm{t}, 1 \mathrm{H}, \mathrm{Ar}, J=7.8 \mathrm{~Hz}), 7.73(\mathrm{~d}, 1 \mathrm{H}, \mathrm{Ar}$, $J=7.6 \mathrm{~Hz}) .{ }^{13} \mathrm{C}-\mathrm{NMR}\left(\mathrm{CDCl}_{3}\right) \delta 15.92\left(\mathrm{CH}_{3}\right), 96.97$ $\left(\mathrm{CH}_{2}\right), 122.05(\mathrm{C}), 124.75(\mathrm{C}), 125.37(\mathrm{CH}), 125.90(\mathrm{CH})$, 130.32 (CH), $131.88(\mathrm{CH}), 132.04(\mathrm{C}), 136.19(\mathrm{C}), 172.47$ (C). IR: $1645 \mathrm{~cm}^{-1}(\mathrm{C}=\mathrm{O})$. LC-MS: $260.0[\mathrm{M}+\mathrm{H}]^{+}$.

\section{General procedure for $5 a-c$ and $6 a-c$}

To a suspension of the substrate of type $\mathbf{1}(\mathbf{1 a}, \mathbf{b})[21,23]$ $(0.54 \mathrm{mmol})$ in anhydrous $\mathrm{CH}_{3} \mathrm{CN}(3 \mathrm{~mL}), 1.08 \mathrm{mmol}$ of $\mathrm{K}_{2} \mathrm{CO}_{3}$ and $0.65 \mathrm{mmol}$ of the appropriate commercially available intermediate $\mathbf{4 a}, \mathbf{b}$ were added. The mixture was stirred at reflux for $8 \mathrm{~h}(\mathbf{5 a}, \mathbf{b}$ and $\mathbf{6 a}, \mathbf{b})$ or for $3 \mathrm{~h}(\mathbf{5 c}-$ and $\mathbf{6 c}$ ). After evaporation of the solvent, ice-cold water $(20 \mathrm{~mL})$ was added, and the suspension was recovered by extraction with ethyl acetate $(3 \times 15 \mathrm{~mL})$. The organic layer was dried over sodium sulfate, and the solvent was evaporated in vacuo to obtain final compounds $\mathbf{5 a}-\mathbf{c}$ and $\mathbf{6 a}-\mathbf{c}$, which were purified by column chromatography using cyclohexane/ethyl acetate 3:1 (for 5a and 6a), 2:1 (for $\mathbf{5 b}$ and $\mathbf{6 b}$ ) or 5:1 (for $\mathbf{5 c}$ and $\mathbf{- 6} \mathbf{c}$ ) as eluents.

\section{(2-Oxo-4-phenylthiazol-3(2H)-yl)methyl benzoate (5a)}

Yield = 39\%; Oil. ${ }^{1} \mathrm{H}-\mathrm{NMR}\left(\mathrm{CDCl}_{3}\right) \delta 5.85\left(\mathrm{~s}, 2 \mathrm{H}, \mathrm{CH}_{2}\right)$, $6.12(\mathrm{~s}, 1 \mathrm{H}, \mathrm{CH}), 7.42-7.48(\mathrm{~m}, 7 \mathrm{H}, \mathrm{Ar}), 7.61(\mathrm{t}, 1 \mathrm{H}$, Ar, $J=7.6 \mathrm{~Hz}), 8.01(\mathrm{~d}, 2 \mathrm{H}, \mathrm{Ar}, J=8.0 \mathrm{~Hz}) .{ }^{13} \mathrm{C}-\mathrm{NMR}$ $\left(\mathrm{CDCl}_{3}\right) \delta 67.08\left(\mathrm{CH}_{2}\right), 99.45(\mathrm{CH}), 128.13(\mathrm{CH}), 128.26$ $(\mathrm{CH}), 128.50(\mathrm{CH}), 128.85(\mathrm{CH}), 129.04(\mathrm{CH}), 130.13(\mathrm{C})$, $130.19(\mathrm{CH}), 130.48(\mathrm{CH}), 136.92(\mathrm{C}), 165.11(\mathrm{C}), 172.78$ (C). IR: $1681 \mathrm{~cm}^{-1}(\mathrm{C}=\mathrm{O}), 1724 \mathrm{~cm}^{-1}(\mathrm{C}=\mathrm{O})$. LC-MS: $312.1[\mathrm{M}+\mathrm{H}]^{+}$.

(5-Acetyl-4-methyl-2-oxothiazol-3(2H)-yl)methyl benzoate (5b) Yield $=21 \% ; \mathrm{mp}=121-123{ }^{\circ} \mathrm{C}(\mathrm{EtOH}) .{ }^{1} \mathrm{H}-\mathrm{NMR}$ $\left(\mathrm{CDCl}_{3}\right) \delta 2.39\left(\mathrm{~s}, 3 \mathrm{H}, \mathrm{CH}_{3}\right), 2.69\left(\mathrm{~s}, 3 \mathrm{H}, \mathrm{COCH}_{3}\right), 6.03$ $\left(\mathrm{s}, 2 \mathrm{H}, \mathrm{CH}_{2}\right), 7.46(\mathrm{t}, 2 \mathrm{H}, \mathrm{Ar}, J=7.8 \mathrm{~Hz}), 7.62(\mathrm{t}, 1 \mathrm{H}$, Ar, $J=7.6 \mathrm{~Hz}$ ), 8.03 (d, $2 \mathrm{H}, \mathrm{Ar}, J=8.4 \mathrm{~Hz}) \cdot{ }^{13} \mathrm{C}-\mathrm{NMR}$ 
$\left(\mathrm{CDCl}_{3}\right) \delta 13.36\left(\mathrm{CH}_{3}\right), 30.33\left(\mathrm{CH}_{3}\right), 65.45\left(\mathrm{CH}_{2}\right), 98.49$ $(\mathrm{CH}), 113.43(\mathrm{C}), 128.15(\mathrm{CH}), 128.50(\mathrm{CH}), 128.61(\mathrm{CH})$, $129.92(\mathrm{CH}), 133.89(\mathrm{CH}), 141.15(\mathrm{C}), 165.10(\mathrm{C}), 169.90$ (C), 189.90 (C). IR: $1643 \mathrm{~cm}^{-1}(\mathrm{C}=\mathrm{O}), 1698 \mathrm{~cm}^{-1}(\mathrm{C}=\mathrm{O})$, $1720 \mathrm{~cm}^{-1}(\mathrm{C}=\mathrm{O})$. LC-MS: $292.0[\mathrm{M}+\mathrm{H}]^{+}$.

\section{3-(3-Methylbenzyl)-4-phenylthiazol-2(3H)-one (5c)}

Yield $=13 \%$; Oil. ${ }^{1} \mathrm{H}-\mathrm{NMR}\left(\mathrm{CDCl}_{3}\right) \delta 2.72\left(\mathrm{~s}, 3 \mathrm{H}, \mathrm{CH}_{3}\right)$, $4.87\left(\mathrm{~s}, 2 \mathrm{H}, \mathrm{CH}_{2}\right), 6.03(\mathrm{~s}, 1 \mathrm{H}, \mathrm{CH}), 6.72-6.78(\mathrm{~m}, 2 \mathrm{H}, \mathrm{Ar})$, $7.03(\mathrm{~d}, 1 \mathrm{H}, \mathrm{Ar}, J=7.6 \mathrm{~Hz}), 7.12(\mathrm{t}, 1 \mathrm{H}, \mathrm{Ar}, J=7.4 \mathrm{~Hz})$, 7.19 (d, $2 \mathrm{H}, \mathrm{Ar}, J=7.0 \mathrm{~Hz}), 7.30-7.40(\mathrm{~m}, 3 \mathrm{H}, \mathrm{Ar}) .{ }^{13} \mathrm{C}-$ $\operatorname{NMR}\left(\mathrm{CDCl}_{3}\right) \delta 21.65\left(\mathrm{CH}_{3}\right), 51.03\left(\mathrm{CH}_{2}\right), 110.54(\mathrm{CH})$, $123.97(\mathrm{CH}), 127.02(\mathrm{CH}), 127.96(\mathrm{CH}), 128.31(\mathrm{CH})$, $128.37(\mathrm{CH}), 128.40(\mathrm{CH}), 128.63(\mathrm{CH}), 128.66(\mathrm{CH})$, $128.89(\mathrm{CH}), 130.70(\mathrm{C}), 131.92(\mathrm{C}), 138.26(\mathrm{C}), 138.33$ (C), 169.90 (C). IR: $1661 \mathrm{~cm}^{-1}(\mathrm{C}=\mathrm{O})$. LC-MS: 282.1 $[\mathrm{M}+\mathrm{H}]^{+}$.

\section{(4-Phenylthiazol-2-yloxy)methyl benzoate (6a)}

Yield $=28 \%$; Oil. ${ }^{1} \mathrm{H}-\mathrm{NMR}\left(\mathrm{CDCl}_{3}\right) \delta 6.41\left(\mathrm{~s}, 2 \mathrm{H}, \mathrm{CH}_{2}\right)$, $6.97(\mathrm{~s}, 1 \mathrm{H}, \mathrm{CH}), 7.34(\mathrm{~d}, 1 \mathrm{H}, \mathrm{Ar}, J=7.8 \mathrm{~Hz}), 7.36-7.44$ $(\mathrm{m}, 4 \mathrm{H}, \mathrm{Ar}), 7.47(\mathrm{t}, 1 \mathrm{H}, \mathrm{Ar}, J=7.8 \mathrm{~Hz}), 7.86(\mathrm{~d}, 2 \mathrm{H}$, Ar, $J=7.8 \mathrm{~Hz}), 8.13(\mathrm{~d}, 2 \mathrm{H}, \mathrm{Ar}, J=8.0 \mathrm{~Hz}) .{ }^{13} \mathrm{C}-\mathrm{NMR}$ $\left(\mathrm{CDCl}_{3}\right) \delta 85.48\left(\mathrm{CH}_{2}\right), 105.95(\mathrm{CH}), 125.94(\mathrm{CH})$, $128.06(\mathrm{CH}), 128.55(\mathrm{CH}), 128.68(\mathrm{CH}), 129.18(\mathrm{CH})$, $130.14(\mathrm{CH}), 133.74(\mathrm{CH}), 134.31(\mathrm{C}), 149.26(\mathrm{C}), 165.26$ (C), 171.56 (C). IR: $1737 \mathrm{~cm}^{-1}(\mathrm{C}=\mathrm{O})$. LC-MS: 312.1 $[\mathrm{M}+\mathrm{H}]^{+}$.

\section{(5-Acetyl-4-methylthiazol-2-yloxy)methyl benzoate (6b)}

Yield = 10\%; Oil. ${ }^{1} \mathrm{H}-\mathrm{NMR}\left(\mathrm{CDCl}_{3}\right) \delta 2.47\left(\mathrm{~s}, 3 \mathrm{H}, \mathrm{CH}_{3}\right)$, $2.64\left(\mathrm{~s}, 3 \mathrm{H}, \mathrm{COCH}_{3}\right), 6.31\left(\mathrm{~s}, 2 \mathrm{H}, \mathrm{CH}_{2}\right), 7.49(\mathrm{t}, 2 \mathrm{H}, \mathrm{Ar}$, $J=7.8 \mathrm{~Hz}), 7.63(\mathrm{t}, 1 \mathrm{H}, \mathrm{Ar}, J=7.4 \mathrm{~Hz}), 8.11(\mathrm{~d}, 2 \mathrm{H}, \mathrm{Ar}$, $J=8.4 \mathrm{~Hz}) .{ }^{13} \mathrm{C}-\mathrm{NMR}\left(\mathrm{CDCl}_{3}\right) \delta 16.86\left(\mathrm{CH}_{3}\right), 26.83$ $\left(\mathrm{CH}_{3}\right), 94.35\left(\mathrm{CH}_{2}\right), 128.60(\mathrm{CH}), 128.63(\mathrm{CH}), 129.95$ $(\mathrm{CH}), 129.99(\mathrm{CH}), 130.10(\mathrm{C}), 133.04(\mathrm{CH}), 143.05$ (C), 153.01 (C), 161.07 (C), 165.90 (C), 196.96 (C). IR: $1695 \mathrm{~cm}^{-1}(\mathrm{C}=\mathrm{O}), 1735 \mathrm{~cm}^{-1}(\mathrm{C}=\mathrm{O})$. LC-MS: 292.0 $[\mathrm{M}+\mathrm{H}]^{+}$.

\section{2-(3-Methylbenzyloxy)-4-phenylthiazole (6c)}

Yield = 13\%; Oil. ${ }^{1} \mathrm{H}-\mathrm{NMR}\left(\mathrm{CDCl}_{3}\right) \delta 2.42\left(\mathrm{~s}, 3 \mathrm{H}, \mathrm{CH}_{3}\right)$, $5.53\left(\mathrm{~s}, 2 \mathrm{H}, \mathrm{CH}_{2}\right), 6.90(\mathrm{~s}, 1 \mathrm{H}, \mathrm{CH}), 7.20-7.25(\mathrm{~m}, 1 \mathrm{H}$, Ar), 7.30-7.35 (m, 4H, Ar), 7.40-7.45 (m, 2H, Ar), 7.88 $(\mathrm{d}, 2 \mathrm{H}, \mathrm{Ar}, J=8.0 \mathrm{~Hz}) .{ }^{13} \mathrm{C}-\mathrm{NMR}\left(\mathrm{CDCl}_{3}\right) \delta 21.65\left(\mathrm{CH}_{3}\right)$, $70.83\left(\mathrm{CH}_{2}\right), 110.84(\mathrm{CH}), 124.17(\mathrm{CH}), 127.52(\mathrm{CH})$, $127.56(\mathrm{CH}), 127.91(\mathrm{CH}), 128.77(\mathrm{CH}), 128.80(\mathrm{CH})$, $129.03(\mathrm{CH}), 129.26(\mathrm{CH}), 129.29(\mathrm{CH}), 133.07(\mathrm{C})$, 138.62 (C), 141.16 (C), 152.33 (C), 154.05 (C). LC-MS: $282.1[\mathrm{M}+\mathrm{H}]^{+}$.

\section{General procedure for 8,9 and $10 a, b$}

$1.02 \mathrm{mmol}$ of $\mathrm{K}_{2} \mathrm{CO}_{3}$ and $0.61 \mathrm{mmol}$ of dibenzyl chloromethyl phosphate (7) [27] were added to a suspension of the substrate 1a-c [21-23] $(0.51 \mathrm{mmol})$ in anhydrous $\mathrm{CH}_{3} \mathrm{CN}(4 \mathrm{~mL})$. The mixture was stirred at reflux for $6 \mathrm{~h}$. After evaporation of the solvent, ice-cold water $(20 \mathrm{~mL})$ was added and the suspension was recovered by extraction with $\mathrm{CH}_{2} \mathrm{Cl}_{2}(3 \times 15 \mathrm{~mL})$. The organic layer was dried on sodium sulfate and evaporated in vacuo to obtain the final compounds, which were purified by column chromatography using cyclohexane/ethyl acetate 1:2 $(\mathbf{8}, \mathbf{9}), 3: 1(\mathbf{1 0 a})$ or hexane/ethyl acetate $3: 1$ for $\mathbf{1 0 b}$, as eluents.

\section{(5-Acetyl-4-methyl-2-oxothiazol-3(2H)-yl)methyl dibenzyl phosphate (8)}

Yield = 12\%; Oil. ${ }^{1} \mathrm{H}-\mathrm{NMR}\left(\mathrm{CDCl}_{3}\right) \delta 2.35\left(\mathrm{~s}, 3 \mathrm{H}, \mathrm{CH}_{3}\right)$, $2.52\left(\mathrm{~s}, 3 \mathrm{H}, \mathrm{COCH}_{3}\right), 5.06\left(\mathrm{~m}, 4 \mathrm{H}, 2 \times \mathrm{CH}_{2} \mathrm{Ph}\right), 5.55(\mathrm{~d}$, $\left.2 \mathrm{H}, \mathrm{CH}_{2} \mathrm{Cl}, J=8.8 \mathrm{~Hz}\right), 7.30-7.40(\mathrm{~m}, 10 \mathrm{H}, \mathrm{Ar}) .{ }^{13} \mathrm{C}-$ $\operatorname{NMR}\left(\mathrm{CDCl}_{3}\right) \delta 13.11\left(\mathrm{CH}_{3}\right), 30.33\left(\mathrm{CH}_{3}\right), 67.17\left(\mathrm{CH}_{2}\right)$, $70.09\left(\mathrm{CH}_{2}\right), 113.59(\mathrm{C}), 128.20(\mathrm{CH}), 128.69(\mathrm{CH})$, $128.85(\mathrm{CH}), 135.18(\mathrm{C}), 140.88(\mathrm{C}), 169.77(\mathrm{C}), 189.75$ (C). IR: $1670 \mathrm{~cm}^{-1}(\mathrm{C}=\mathrm{O}), 1701 \mathrm{~cm}^{-1}(\mathrm{C}=\mathrm{O}) . \mathrm{LC}-\mathrm{MS}$ : $448.0[\mathrm{M}+\mathrm{H}]^{+}$.

\section{[(5-Acetyl-4-methylthiazol-2-yloxy)methyl] dibenzyl phosphate} (9)

Yield $=13 \%$; Oil. ${ }^{1} \mathrm{H}-\mathrm{NMR}\left(\mathrm{CDCl}_{3}\right) \delta 2.45\left(\mathrm{~s}, 3 \mathrm{H}, \mathrm{CH}_{3}\right)$, $2.58\left(\mathrm{~s}, 3 \mathrm{H}, \mathrm{COCH}_{3}\right), 5.09\left(\mathrm{~d}, 4 \mathrm{H}, 2 \times \mathrm{CH}_{2} \mathrm{Ph}, J=8.0 \mathrm{~Hz}\right)$, $5.92\left(\mathrm{~d}, 2 \mathrm{H}, \mathrm{CH}_{2} \mathrm{Cl}, J=14.4 \mathrm{~Hz}\right), 7.30-7.40(\mathrm{~m}, 10 \mathrm{H}, \mathrm{Ar})$. ${ }^{13} \mathrm{C}$-NMR $\left(\mathrm{CDCl}_{3}\right) \delta 18.47\left(\mathrm{CH}_{3}\right), 30.13\left(\mathrm{CH}_{3}\right), 69.78$ $\left(\mathrm{CH}_{2}\right), 88.12\left(\mathrm{CH}_{2}\right), 127.93(\mathrm{CH}), 128.62(\mathrm{CH}), 128.70$ $(\mathrm{CH}), 129.21(\mathrm{CH}), 135.30(\mathrm{C}), 154.47(\mathrm{C}), 190.15(\mathrm{C})$. IR: $1653 \mathrm{~cm}^{-1}(\mathrm{C}=\mathrm{O})$. LC-MS: $448.0[\mathrm{M}+\mathrm{H}]^{+}$.

Dibenzyl [(4-phenylthiazol-2-yloxy)methyl] phosphate (10a) Yield $=18 \%$; Oil. ${ }^{1} \mathrm{H}-\mathrm{NMR}\left(\mathrm{CDCl}_{3}\right) \delta 5.10(\mathrm{~d}, 4 \mathrm{H}$, $\left.2 \times \mathrm{CH}_{2} \mathrm{Ph}, J=8.0 \mathrm{~Hz}\right), 6.04\left(\mathrm{~d}, 2 \mathrm{H}, \mathrm{CH}_{2} \mathrm{Cl}, J=13.6 \mathrm{~Hz}\right)$, $6.96(\mathrm{~s}, 1 \mathrm{H}, \mathrm{CH}), 7.31-7.43(\mathrm{~m}, 13 \mathrm{H}, \mathrm{Ar}), 7.84(\mathrm{~d}, 2 \mathrm{H}$, Ar, $J=7.6 \mathrm{~Hz}) \cdot{ }^{13} \mathrm{C}-\mathrm{NMR}\left(\mathrm{CDCl}_{3}\right) \delta 69.71\left(\mathrm{CH}_{2}\right), 88.50$ $\left(\mathrm{CH}_{2}\right), 106.13(\mathrm{CH}), 125.95(\mathrm{CH}), 127.94(\mathrm{CH}), 128.11$ $(\mathrm{CH}), 128.59(\mathrm{CH}), 128.66(\mathrm{CH}), 134.14(\mathrm{C}), 135.45(\mathrm{C})$, $149.29(\mathrm{C}), 171.00(\mathrm{C})$. LC-MS: $468.2[\mathrm{M}+\mathrm{H}]^{+}$.

\section{Dibenzyl [(4-methylthiazol-2-yloxy)methyl] phosphate (10b)}

Yield = 15\%; Oil. ${ }^{1} \mathrm{H}-\mathrm{NMR}\left(\mathrm{CDCl}_{3}\right) \delta 2.27\left(\mathrm{~s}, 3 \mathrm{H}, \mathrm{CH}_{3}\right)$, $5.09\left(\mathrm{~d}, 4 \mathrm{H}, 2 \times \mathrm{CH}_{2} \mathrm{Ph}, J=7.6 \mathrm{~Hz}\right), 5.92\left(\mathrm{~d}, 2 \mathrm{H}, \mathrm{CH}_{2} \mathrm{Cl}\right.$, $J=14.0 \mathrm{~Hz}), 6.33(\mathrm{~s}, 1 \mathrm{H}, \mathrm{CH}), 7.30-7.40(\mathrm{~m}, 10 \mathrm{H}, \mathrm{Ar})$. ${ }^{13} \mathrm{C}$-NMR $\left(\mathrm{CDCl}_{3}\right) \delta 17.10\left(\mathrm{CH}_{3}\right), 69.41\left(\mathrm{CH}_{2}\right), 93.80$ $\left(\mathrm{CH}_{2}\right), 114.93(\mathrm{CH}), 127.15(\mathrm{CH}), 127.64(\mathrm{CH}), 128.91$ 
(CH), 135.44 (C), 149.69 (C), 153.01 (C). LC-MS: 406.1 $[\mathrm{M}+\mathrm{H}]^{+}$.

\section{HNE inhibition assay}

Compounds were dissolved in 100\% DMSO at $5 \mathrm{mM}$ stock concentrations. The final concentration of DMSO in the reactions was $1 \%$, and this level of DMSO had no effect on enzyme activity. The HNE inhibition assay was performed in black flat-bottom 96-well microtiter plates. Briefly, a buffer solution containing $200 \mathrm{mM}$ Tris- $\mathrm{HCl}$, $\mathrm{pH} 7.5,0.01 \%$ bovine serum albumin, and $0.05 \%$ Tween20 and $20 \mathrm{mU} / \mathrm{mL}$ of HNE (Calbiochem) was added to wells containing different concentrations of each compound. The reaction was initiated by addition of $25 \mu \mathrm{M}$ elastase substrate ( $N$-methylsuccinyl-Ala-Ala-Pro-Val7-amino-methyl-coumarin, Calbiochem) in a final reaction volume of $100 \mu \mathrm{L} /$ well. Kinetic measurements were obtained every $30 \mathrm{~s}$ for $10 \mathrm{~min}$ at $25^{\circ} \mathrm{C}$ using a Fluoroskan Ascent FL fluorescence microplate reader (Thermo Electron, MA) with excitation and emission wavelengths at 355 and $460 \mathrm{~nm}$, respectively.

\section{Instrumental}

The LC-MS/MS analysis was carried out using a Varian 1200L triple quadrupole system (Palo Alto, CA, USA) equipped with two Prostar 210 pumps, a Prostar 410 auto sampler, and an electrospray source (ESI) operating in the positive ion mode. The ion sources and ion optics parameters were optimized and $1 \mu \mathrm{g} / \mathrm{mL}$ working solution of the test compounds was injected via syringe pump at $10 \mu \mathrm{L} / \mathrm{min}$. Raw data were collected and processed by Varian Workstation Version 6.8 software.

\section{Standard solutions}

Stock solutions of analytes were prepared in acetonitrile at $1.0 \mathrm{mg} / \mathrm{mL}$ and stored at $4{ }^{\circ} \mathrm{C}$. Working solutions of each analyte were freshly prepared by diluting stock solutions in acetonitrile up to a concentration of $1.0 \mu \mathrm{g} / \mathrm{mL}$.

\section{Tandem mass spectrometry experiments}

Compounds 8 and $\mathbf{9}$ are positional isomers and under ESI-MS conditions showed the production of abundant protonated molecules, detected at the same $m / z$ value, and only a few fragment ions at very low relative abundance (less than $2 \%$ ). Therefore, we utilized specific collisionally activated MS/MS decomposition pathways to assign their structures. Accordingly, a series of MS/MS experiments were performed by applying different approaches, based on the potential of a triple quadrupole system.
Energy resolved tandem mass spectrometry (ERMS) experiments [28] were performed to evaluate the collision activated decomposition pathway of isomers 8 and 9. The ERMS experiments involve a series of product ion scan spectra acquisitions, which were obtained by increasing the collision energy stepwise in the range from 0 to $20 \mathrm{~V}$. The results obtained were used to study the fragmentation of molecular species from each analyte and build its breakdown curves. In detail, the product ion scan spectra were acquired in the $\mathrm{m} / \mathrm{z}$ range from 50 to 650 , scan time $600 \mathrm{~ms}$, and argon was used as collisional gas. The breakdown curves data were obtained by introducing $1.0 \mu \mathrm{g} / \mathrm{mL}$ of each analyte via syringe pump at $10 \mu \mathrm{L} / \mathrm{min}$; the protonated molecule was isolated and the abundance of product ions were monitored. The values of the relative intensities of MS/MS spectra used to build the breakdown curves were the mean of 50 scans for each collision energy and the collision gas pressure was critically evaluated in order to achieve valid and reproducible fragmentation patterns on the basis of the results obtained [29].

\section{Docking analysis}

A 3-D model of compound $2 \mathbf{e}$ was built and pre-optimized by Chem3D (version 12.0.2) software (ChemBioOffice 2010 Suite). The model was imported into the Molegro Virtual Docker (MVD) program together with the structure of human neutrophil elastase (1HNE entry of Protein Data Bank), where the enzyme is complexed with peptide chloromethyl ketone inhibitor [30]. All water molecules were deleted from the protein model. The search area for docking poses was defined as a sphere with $10 \AA$ radius centered at the nitrogen atom in the five-membered ring of the co-crystallized peptide. Side chains of 42 residues in the vicinity of the binding site were set flexible during docking, as described previously [16]. The HNE catalytic triad comprised of Ser195, His57, and Asp102 was also among the residues with flexible side chains. Simulation of the receptor flexibility was performed with the standard technique built in the Molegro program (Molegro Virtual Docker. User Manual, 2010). Values of 0.9 and 0.7 , respectively, were assigned to the "Tolerance" and "Strength" parameters of the MVD "Side chain Flexibility" wizard. Forty docking runs were performed with full flexibility of ligand $\mathbf{2 e}$ around its rotatable bonds. Geometric parameters of the lowest-energy docking pose were determined with measurement tools of MVD software in order to estimate the ability of compound $2 \mathbf{e}$ to form a Michaelis complex between the 
hydroxyl group of Ser195 and each carbonyl group of the ligand, according to previously reported methods [24, 25].

\section{Additional file}

Additional file 1: Table S1. Elemental analysis.

\section{Authors' contributions}

$L C, A l, G G$, and $C V$ performed the synthesis. MM performed the mass spectrometry experiments. IAS performed the biological tests. MG and MTQ conceived and designed the study. AlK carried out molecular modelling experiments. LC, GB, AC, MG, IAS, AIK, and MTQ wrote and revised the manuscript. All authors read and approved the final manuscript.

\section{Author details}

1 Sezione di Farmaceutica e Nutraceutica, NEUROFARBA, Università degli Studi di Firenze, Via Ugo Schiff 6, 50019 Sesto Fiorentino, Firenze, Italy. ${ }^{2}$ Institute of Pharmaceutical Science, King's College London, 150 Stamford Street, London SE1 9NH, UK. ${ }^{3}$ Department of Microbiology and Immunology, Montana State University, Bozeman, MT 59717, USA. ${ }^{4}$ Department of Biotechnology and Organic Chemistry, Tomsk Polytechnic University, Tomsk 634050, Russia. ${ }^{5}$ Scientific Research Institute of Biological Medicine, Altai State University, Barnaul 656049, Russia.

\section{Acknowledgements}

This research was supported in part by National Institutes of Health IDeA Program COBRE Grant GM1 10732; USDA National Institute of Food and Agriculture Hatch Project 1009546; the Montana State University Agricultural Experiment Station; and Tomsk Polytechnic University Competitiveness Enhancement Program Grant, Project TPU CEP_IHTP_73\2017.

\section{Competing interests}

The authors declare that they have no competing interests.

\section{Availability of data and materials}

All data are fully available without restriction at the author's Institutions.

\section{Consent for publication}

Not applicable.

\section{Ethics approval and consent to participate}

Not applicable.

\section{Funding}

National Institutes of Health IDeA Program COBRE Grant GM110732 USDA National Institute of Food and Agriculture Hatch Project 1009546 Tomsk Polytechnic University Competitiveness Enhancement Program Grant, Project TPU CEP_IHTP_73\2017.

\section{Publisher's Note}

Springer Nature remains neutral with regard to jurisdictional claims in published maps and institutional affiliations.

Received: 12 September 2017 Accepted: 26 November 2017 Published online: 06 December 2017

\section{References}

1. Korkmaz B, Horwitz MS, Jenne DE, Gauthier F (2010) Neutrophil elastase, proteinase 3, and cathepsin $\mathrm{G}$ as therapeutic targets in human diseases. Pharmacol Rev 62:726-759

2. Pham CT (2006) Neutrophil serine proteases: specific regulators of inflammation. Nat Rev Immunol 6:541-550

3. Fitch PM, Roghanian A, Howie SE, Sallenave JM (2006) Human neutrophil elastase inhibitors in innate and adaptive immunity. Biochem Soc Trans 34:279-282

4. Kelly E, Greene MC, McElvanev N (2008) Targeting neutrophil elastase in cystic fibrosis. Expert Opin Ther Targets 12:145-157

5. Korkmaz B, Moreau T, Gauthier F (2008) Neutrophil elastase, proteinase 3 and cathepsin G: physicochemical properties, activity and physiopathological functions. Biochimie 90:227-242

6. Mannino DM (2005) Epidemiology and global impact of chronic obstructive pulmonary disease. Semin Respir Crit Care Med 26:204-210

7. Tsushima K, King SL, Aggarwal NR, De Gorordo A, D’Alessio FR, Kubo K (2009) Acute lung injury review. Intern Med 48:621-630

8. Voynow JA, Fischer BM, Zheng S (2008) Proteases and cystic fibrosis. Int J Biochem Cell Biol 40:1238-1245

9. Iwata K, Doi A, Ohji G, Oka H, Oba Y, Takimoto K, Igarashi W, Gremillion DH, Shimada T (2010) Effect of neutrophil elastase inhibitor (sivelestat sodium) in the treatment of acute lung injury (ALI) and acute respiratory distress syndrome (ARDS): a systematic review and meta-analysis. Intern Med 49(22):2423-2432

10. Tebbutt SJ (2000) Technology evaluation: transgenic alpha-1-antitrypsin (AAT), PPL therapeutics. Curr Opin Mol Ther 2:199-204

11. Stockley R, De Soyza A, Gunawardena K, Perret J, Forsman-Semb K, Entwistle N, Snell N (2013) Phase II study of a neutrophil elastase inhibitor (AZD9668) in patients with bronchiectasis. Respir Med 107(4):524-533

12. Vogelmeier C, Aquino TO, O'Brein CD, Perret J, Gunawardena K (2012) A randomised, placebo-controlled, dose-finding study of AZD9668, an oral inhibitor of neutrophil elastase, in patients with chronic obstructive pulmonary disease treated with tiotropium. COPD 9(2):111-120

13. Von Nussbaum F, Li VMJ, Allerheiligen S, Anlauf S, Bärfacker L, Bechem M, Delbeck M, Fitzgerald MF, Gerisch M, Gielen-Haertwig H, Haning $H$, Karthaus D, Lang D, Lustig K, Meibom D, Mittendorf J, Rosentreter U, Schäfer M, Schäfer S, Schamberger J, Telan LA, Tersteegen A (2015) Freezing the bioactive conformation to boost potency: the identification of BAY 85-8501, a selective and potent inhibitor of human neutrophi elastase for pulmonary diseases. ChemMedChem 10(7):1163-1173

14. Crocetti L, Giovannoni MP, Schepetkin IA, Quinn MT, Khlebnikov Al, Cilibrizzi A, Dal Piaz V, Graziano A, Vergelli C (2011) Design, synthesis and evaluation of $\mathrm{N}$-benzoylindazole derivatives and analogues as inhibitors of human neutrophil elastase. Bioorg Med Chem 19:4460-4472

15. Crocetti L, Schepetkin IA, Ciciani G, Giovannoni MP, Guerrini G, lacovone A, Khlebnikov Al, Kirpotina LN, Quinn MT, Vergelli C (2016) Synthesis and pharmacological evaluation of indole derivatives as deaza analogues of potent human neutrophil elastase inhibitors. Drug Dev Res 77(6):285-299

16. Crocetti L, Schepetkin IA, Cilibrizzi A, Graziano A, Vergelli C, Giomi D, Khlebnikov Al, Quinn MT, Giovannoni MP (2013) Optimization of $\mathrm{N}$-benzoylindazole derivatives as inhibitors of human neutrophil elastase. J Med Chem 56:6259-6272

17. Giovannoni MP, Schepetkin IA, Crocetti L, Ciciani G, Cilibrizzi A, Guerrini G, Khlebnikov Al, Quinn MT, Vergelli C (2016) Cinnoline derivatives as human neutrophil elastase inhibitors. J Enzym Inhib Med Chem 31(4):628-639

18. Vergelli C, Schepetkin IA, Crocetti L, lacovone A, Giovannoni MP, Guerrini G, Khlebnikov Al, Ciattini S, Ciciani G, Quinn MT (2017) Isoxazol-5(2H)-one: a new scaffold for potent human neutrophil elastase (HNE) inhibitors. $J$ Enzym Inhib Med Chem. https://doi.org/10.1080/14756366.2017.1326915

19. Wood G, Duncan KW, Meades C, Gibson D, Mclachlan JC, Perry A, Blake D, Zheleva DI, Fischer PM (2005) Pyrimidin-4-yl-3,4-thione compounds and their use in therapy. PCT Int Appl. WO 2005/042525 A1 
20. Yarligan S, Ogretir C, Csizmadia IG, Acikkalp E, Berber H, Arslan T (2005) An ab initio study on protonation of some substituted thiazole derivates. J Mol Struct Theochem 715:199-203

21. Biancalani C, Giovannoni MP, Pieretti S, Cesari N, Graziano A, Vergelli C, Cilibrizzi A, Di Gianuario A, Colucci MA, Mangano G, Garrone B, Polenzani L, Dal Piaz V (2009) Further studies on arylpiperazinyl alkyl pyridazinones: discovery of an exceptionally potent, orally active, antinociceptive agent in thermally induced pain. J Med Chem 52(23):7397-7409

22. Kikelj D, Urleb U (2002) Product class 17: thiazoles. Sci Synth 11:627-833

23. Pihlaja K, Ovcharenko V, Kohlemainen E, Laihia K, Fabian-Walter MF, Dehne H, Perjessy A, Kleist M, Teller J, Šustekovà Z (2002) A correlation IR, $\mathrm{MS},{ }^{1} \mathrm{H},{ }^{13} \mathrm{C}$ and ${ }^{15} \mathrm{~N}$ NMR and theoretical study of 4-arylthiazol-2(3H)-one. J Chem Soc Perkin Trans 2:329-336

24. Burgi HB, Dunitz JD, Lehn JM, Wipff G (1974) Stereochemistry of reaction paths at carbonyl centers. Tetrahedron 30:1563-1572

25. Vergely I, Laugaa P, Reboud-Ravaux M (1996) Interaction of human leukocyte elastase with a $\mathrm{N}$-aryl azetidinone suicide substrate: conformational analyses based on the mechanism of action of serine proteinases. J Mol Graph 14(145):158-167
26. Sieńczyk M, Winiarski L, Kasperkiewicz P, Psurski M, Wietrzyk J, Oleksyszyn J (2011) Simple phosphonic inhibitors of human neutrophil elastase. Bioorg Med Chem Lett 21:1310-1314

27. Mäntylä A, Vepsäläinen J, Järvinen T, Nevalainen T (2002) A novel synthetic route for the preparation of alkyl and benzyl chloromethyl phosphates. Tetrahedron Lett 43:3793-3794

28. Menicatti M, Guandalini L, Dei S, Floriddia E, Teodori E, Traldi P, Bartolucci $G$ (2016) Energy resolved tandem mass spectrometry experiments for resolution of isobaric compounds: a case of cis/trans isomerism. Eur J Mass Spectrom 22(5):235-243

29. Menicatti M, Guandalini L, Dei S, Floriddia E, Teodori E, Traldi P, Bartolucci G (2016) The power of energy-resolved tandem mass spectrometry experiments for resolution of isomers: the case of drug plasma stability investigation of multidrug resistance inhibitors. Rapid Commun Mass Spectrom 30:423-432

30. Navia MA, McKeever BM, Springer JP, Lin TY, Williams HR, Fluder EM, Dorn CP, Hoogsteen K (1989) Structure of human neutrophil elastase in complex with a peptide chloromethyl ketone inhibitor at $1.84 \AA$ resolution. Proc Natl Acad Sci USA 86:7-11

\section{Submit your manuscript to a SpringerOpen ${ }^{\odot}$ journal and benefit from:}

- Convenient online submission

- Rigorous peer review

- Open access: articles freely available online

- High visibility within the field

- Retaining the copyright to your article

Submit your next manuscript at $\gg$ springeropen.com 